\title{
Impact of explosive volcanic eruptions on the main climate variability modes
}

\author{
Didier Swingedouw ${ }^{1}$, Juliette Mignot ${ }^{2}$, Pablo Ortega ${ }^{3}$, Myriam Khodri ${ }^{2}$, Martin Menegoz ${ }^{4}$, \\ Christophe Cassou ${ }^{5}$ and Vincent Hanquiez ${ }^{1}$ \\ ${ }^{1}$ Environnements et Paléoenvironnements Océaniques et Continentaux (EPOC), UMR CNRS 5805 \\ EPOC-OASU-Université de Bordeaux, Allée Geoffroy Saint-Hilaire, Pessac 33615, France. \\ ${ }^{2}$ Sorbonne Universités (UPMC, Univ. Paris 06)-CNRS-IRD-MNHN, LOCEAN Laboratory, 4 place \\ Jussieu, F-75005 Paris, France. \\ ${ }^{3}$ NCAS-Climate, University of Reading, UK \\ ${ }^{4}$ Barcelona Supercomputing Center, Edifici Nexus II, C/ Jordi Girona, 31, 08034 Barcelona, Spain. \\ ${ }^{5}$ UMR CNRS 5318 CECI - CERFACS, 42 Avenue G. Coriolis, 31057 Toulouse, France.
}

\begin{abstract}
Volcanic eruptions eject large amounts of materials into the atmosphere, which can have an impact on climate. In particular, the sulphur dioxide gas released in the stratosphere leads to aerosol formation that reflects part of the incoming solar radiation, thereby affecting the climate energy balance. In this review paper, we analyse the regional climate imprints of large tropical volcanic explosive eruptions. For this purpose, we focus on the impact on three major climatic modes, located in the Atlantic (the North Atlantic Oscillation: NAO and the Atlantic Multidecadal Oscillation: AMO) and Pacific (the El Niño Southern Oscillation, ENSO) sectors. We present an overview of the chain of events that contributes to modifying the temporal variability of these modes. Our literature review is complemented by new analyses based on observations of the instrumental era as well as on available proxy records and climate model simulations that cover the last millennium. We show that the impact of volcanic eruptions of the same magnitude or weaker than $1991 \mathrm{Mt}$. Pinatubo eruption on the NAO and ENSO is hard to detect, due to the noise from natural climate variability. There is however a clear impact of the direct radiative forcing resulting from tropical eruptions on the AMO index both in reconstructions and climate model simulations of the last millennium, while the impact on the ocean circulation remains model-dependent. To increase the signal to noise ratio and better evaluate the climate response to volcanic eruptions, improved reconstructions of these climatic modes and of the radiative effect of volcanic eruptions are required on a longer time frame than the instrumental era. Finally, we evaluate climate models' capabilities to reproduce the observed and anticipated impacts and mechanisms associated with volcanic forcing, and assess their potential for seasonal to decadal prediction. We find a very large spread in the simulated responses across the different climate models. Dedicated experimental designs and analyses are therefore needed to decipher the cause for this large uncertainty.
\end{abstract}

\section{1) Introduction}

Volcanic eruptions are unpredictable natural events that can induce large casualties because of lava and other pyroclastic effects. In historical times, three volcanic eruptions have deeply affected Europe and therefore received a lot of interest. The first one is related to the volcano Thera located in the eastern Mediterranean. Its eruption around 3,600 years ago is among the largest of the Holocene period, devastating the local settlements of the Minoan civilisation. The second one is the well-known Vesuvius eruption (79 AD), responsible for the destruction of Pompeii. More recently, the Laki eruption in 1783 in Iceland led to large casualties all over Europe due to a poisonous thick haze, rich in sulphur dioxide, that travelled in the continent and 
strongly increased mortality. The largest volcanic eruption during the last millennium (in 1257) was from Samalas, located in Indonesia (Lavigne et al. 2013). This eruption is thought to have ejected about 10 times more material in the atmosphere (aerosols and particles) than the Vesuvius eruption. The latest large eruption is Mount Pinatubo, which is located in the Philippines and erupted in June 1991 (with comparable volume of ejected material to the Vesuvius eruption). It is a very important case study for understanding the impact of volcanic eruptions on climate as it is the sole large eruption for which large-scale high quality observations of the atmosphere are available, in particular thanks to remote sensing.

Formation of volcanoes is related to tectonic activity (subduction or divergence of lithospheric plates), or to the presence of a hot spot in the terrestrial mantle that pierces the crust. Magma accumulates in a so-called magma chamber, a large underground pool of liquid rock found beneath the volcano. The related rise of internal pressure allows the gases, mainly composed of sulphur dioxide $\left(\mathrm{SO}_{2}\right)$, water vapour $\left(\mathrm{H}_{2} \mathrm{O}\right)$ and carbon dioxide $\left(\mathrm{CO}_{2}\right)$, to separate from the lava. Due to their high buoyancy, these gases push vertically towards the surface and the magma reservoir inflates. At a certain point, the pressure is too high and the crust breaks, sometimes resulting in very violent eruptions. The gases entraining magmatic materials, called tephra (from the Greek meaning "ash"), are the first to emerge at the surface. A large amount of dust, sulphur dioxide, water vapour, and carbon dioxide gases are then released into the atmosphere within the plume of the eruption (Figure 1). It is through this release of gases (in particular of sulphur dioxide) into the atmosphere that volcanic eruptions can have a worldwide impact on climate. Their most direct effect is a modification of the scattering of the incoming short wave radiation from the sun, which leads to a change in the opacity of the atmosphere and in the perception of the colour of the sky. For instance, it has been hypothesized that the painting "The Scream" by Munch realised in 1893, with a reddish sky in Oslo, could be related to the impact of Krakatau volcano erupting in 1883 in Indonesia.

Different types of eruptions exist (cf. Table 1): they could be explosive (Pinatubo, injecting into the stratosphere up to $20 \mathrm{Tg}$ of $\mathrm{SO}_{2}$ in 1 day) or flood basalts/effusive (Bardarbunga in Iceland injecting 6-20 $\mathrm{Tg}$ of $\mathrm{SO}_{2}$ in the troposphere in 6 months, Etna in Sicilia, $20 \mathrm{Tg}$ of $\mathrm{SO}_{2}$ in 10 years). In this review, we will mainly focus on substantial explosive eruptions, due to their larger climate impacts.

To measure volcanic eruption magnitude, different indices have been proposed: e.g., Sapper (1927) index, Dust Veil Index (DVI, Lamb 1970), volcanic explosivity index (VEI, Newhall \& Self 1982), Volcanic Sulphur Dioxide (VSI, Schnetzler et al. 1997) and Ice Core Volcanic Index (IVI, Gao et al. 2008). One of the most used is the VEI. It is a composite estimate of the explosive character of an eruption (Newhall \& Self 1982), which is related with the volume of tephra ejected from the magma chamber towards the atmosphere. It ranks volcanic events from 0 for non-explosive eruptions to 8 for the largest eruptions registered. The logarithmic scale is mainly based on the amount of material that eruptions expel. Table 1 depicts the main characteristics of the different type of volcanic eruptions, with information on their duration, the height of their explosive column and the altitude of injection of tephra and gazes. The VEI scale is almost linearly related to VSI as shown in Schnetzler et al. (1997), indicating that these two scales are essentially compatible. However, while this latter index is widely used in the general literature on volcanic eruptions, it is not suitable for climate-related studies. Indeed, the IVI is more adequate as it reflects more closely the radiative impact of the eruptions. This index is a measure of the sulphate aerosol deposition in ice cores, which can be inversed to infer the quantity of these aerosols present in the atmosphere at the time of the eruption, providing thus a more direct estimate of the changes in optical depth and radiative properties of the atmosphere (Gao et al. 2008). In this review paper, we will focus on explosive eruptions, characterised by an IVI index among the 20 largest of the last millennium (e.g. Mt. Pinatubo, Samalas). These generally coincide with eruptions whose VEI ranges from 5 to 7.

Following an eruption, the sulphur dioxide released into the atmosphere is further oxidized into sulphate aerosols $\left(\mathrm{H}_{2} \mathrm{SO}_{4}\right)$. Depending on the height of the plumes, these can strongly affect the optical depth of the considered atmospheric layer, leading to an important radiative impact on the incoming solar radiation (Robock 2000; Timmreck 2012), as described 
above. When limited to the troposphere (below 10-20 km altitude), many of these aerosols are used as nuclei to form droplets in clouds, which favour precipitation leading to a rapid washout of the aerosols. In this case, the volcanic eruptions have a weak, local and short-term impact on climate. When the volcanic plume reaches the stratosphere, which is much drier than the troposphere and thereby precluding any washout, the formed aerosols have a longer lifetime (efolding residence time of about 1 year, Robock 2000), leading to radiative effect up to 2 or 3 years for large volcanic eruptions. They produce a cooling of the Earth's surface by reflecting a part of the incoming solar radiation (parasol effect) but a warming of the stratosphere, through absorption of both solar radiation in the near-infrared spectral band and upward long wave radiation. The aerosol loading into the stratosphere after a volcanic eruption is in general not uniform spatially. For extra-tropical eruptions, the aerosol formation, transport and sedimentation (and therefore the climate impact) are almost exclusively restricted to the surrounding areas, up to the hemispheric scale for very large eruptions like the Laki's one in 1783. By contrast, when the eruption takes place in the tropics, as it was the case for Samalas in 1257 or Mt. Pinatubo in 1991, the aerosols are transported in both hemispheres through the main atmospheric streams of the so-called Brewer-Dobson circulation in the stratosphere (cf. Vernier et al. 2009). To illustrate this, the observed propagation of the aerosols from the Mt. Pinatubo volcanic eruption is represented in Figure 2, depicting the variations of the aerosol optical depth for the $550 \mathrm{~nm}$ wavelength in latitude and altitude as a function of time in the 1990s. In this review paper, we will only consider the largest volcanic explosive eruptions over the last millennium that occurred in the tropical area and therefore rapidly and significantly affected both hemispheres.

Because of heterogeneous spatial patterns and possible feedbacks on several elements of the climate system, these volcanic eruptions not only induce a global cooling of the surface climate of the Earth by altering the incoming solar radiation, but may also affect the climate system variability at regional scale. This variability is typically organised into large-scale patterns and in this review, we will focus on three of the most well-known climate variability modes, with large impacts on human activities: the North Atlantic Oscillation (NAO), the El-Niño Southern Oscillation (ENSO) and the Atlantic Multidecadal Oscillation (AMO).

The NAO is the main mode of sea-level pressure variability over the North Atlantic sector (Hurrell 1994) from daily to decadal timescales, thereby strongly impacting the low-level atmospheric circulation and in particular the European climate in winter. It can thus be defined as the first mode obtained from a principal component analysis of winter sea-level pressure, or alternatively as the standardised station-based difference in pressure between the Azores and Iceland, where the main centres of action are located (Hurrell 1994). The NAO is also associated with a larger scale mode of variability called Arctic Oscillation (AO), which interacts with the polar vortex. This latter is centred over the pole and is related to the radiative cooling during the winter polar night. This cooling leads to very strong meridional temperature gradient and through thermal wind balance, strong westerly winds ( $c f$. Figure 1), which can exceed $180 \mathrm{~m} / \mathrm{s}$. It is now well known that interactions between the stratosphere and the troposphere through downward propagative waves can strongly affect the NAO phase ( $c f$. Gerber et al. 2012).

The two other selected modes are more related with the Earth's surface and have very strong impacts on climate from interannual to decadal timescales. It is thus crucial to understand their underlying mechanisms in detail as well as their sensitivity to forcings that are external to the climate system, such as volcanic eruptions.

ENSO is a coupled ocean-atmosphere variability mode which has its origin in the tropical Pacific (Bjerknes 1969). It is the dominant mode of variability over the globe and has worldwide climate impacts (Vecchi \& Wittenberg 2010). In its positive phase, also called El Niño, the mode is associated with positive SST anomalies in the tropical East Pacific (cf. Figure 1) and anomalously weak trade winds over the Pacific basin. The negative phase, called La Niña, corresponds to cold anomalies in the East Pacific, associated to strengthened upwelling and strong trade winds. These different events lead to the modification of atmospheric tropical convection and large-scale circulation (Larkin \& Harrison 2005) that remotely affects large areas of the planet through so-called teleconnections (Wallace \& Gutzler 1981). Historically, the 
Southern Oscillation Index has been defined as the difference of pressure between Tahiti (central Pacific) and Darwin (Australia), both strongly related to tropical Pacific SST variability via Bjerknes feedback (Philander 1999). Alternatively, ENSO variations can also be defined by sea surface temperature (SST) variations in specific regions of the tropical Pacific (Trenberth \& Stepaniak 2001).

The AMO refers to a basin-wide fluctuation of the SST in the North Atlantic observed at decadal timescales. It is defined as the average SST over the North Atlantic $\left(0-60^{\circ} \mathrm{N}\right)$, from which the signal of external forcing can be removed through different techniques (Trenberth \& Shea 2006). It can be alternatively isolated through principal component analysis of SSTs over the North Atlantic sector (Delworth et al. 2007). The variations of the AMO have been shown to promote specific climatic conditions. For instance, during its positive phase there is an increasing number of droughts over North America (Sutton \& Hodson 2005) and mid-latitude hurricanes in the Atlantic (Trenberth \& Shea 2006). It also induces low frequency modulations of the Sahelian (Martin \& Thorncroft 2014; Zhang \& Delworth 2006) and Indian (Zhang \& Delworth 2006) monsoon systems, as well as the decadal variability in the Pacific (Zhang \& Delworth 2007, McGregor et al. 2014).

Over the instrumental era (starting around 1850), only five major eruptions (Krakatau, Santa María, Agung, El Chichón and Pinatubo) have occurred around the world. The sampling is therefore too small to make robust statistical analyses (e.g. with a high signal to noise ratio) to fully address the associated climate impacts, given the chaotic nature and stochastic noise inherent to the climate system, leading to strong internal variability. To better understand the fingerprints of volcanic eruptions on climate, larger samples coming from climate reconstructions of the last thousand years prove to be very useful. Proxy data of different kinds (tree ring data, speleothems, sediment and ice cores...) have been collected for several decades and provide an imperfect but useful view of the climate variations over this time period. In addition, a dynamical representation of its complexity is necessary to understand thoroughly the processes at play in the climate system. Numerical climate modelling provides this.

Climate models have been developed in the last few decades to improve our understanding of climate dynamics and also with the aim of predicting future variations of the climatic modes. Meteorology is a well-known aspect of the prediction side; it is based on the use of stand-alone atmospheric numerical models, based on primitive equations of the motion of fluid dynamics. This type of numerical models is called General Circulation Model (GCM). At longer timescales, other components of the climate system have to be taken into account for an accurate representation of the evolution of this system. Coupled atmosphere-ocean general circulation models (AOGCMs) have thus been developed to reproduce, understand and forecast the future variations of the climate system from the seasonal to centennial time scale. Nowadays more than 20 institutes are developing such complex climate models around the world. Projections of these models based on scenarios of evolution of human activities provide the experimental basis for the Intergovernmental Panel on Climate Change (IPCC) reports to assess the possible outcomes for the future climate. Other simulations are also performed to evaluate the performance of these models in reproducing the past variations of the climate, for instance over the last 1000 years (last millennium). All these simulations are stored and shared within the Coupled Model Intercomparison Project (CMIP), whose last exercise is denominated CMIP5. Last millennium simulations are part of the so-called PMIP3 subproject, dedicated to paleoclimate modelling.

In the present review paper, we analyse in detail the impact of volcanic eruptions on climate variability at seasonal to decadal time scales (cf. Figure 1). More specifically, we describe the current knowledge regarding the following questions:

- What is the potential impact of a volcanic eruption on the main modes of observed climate variability? How are they affected and for how long?

- What are the processes associated with such an impact?

- Are such impacts robustly represented in models?

We first describe how volcanic eruptions modify the radiative budget of the Earth and therefore its global temperature. Then, we analyse the impact of eruptions on the NAO, the ENSO 
and finally the AMO using both observations and climate model simulations. General discussions, conclusions and perspectives end the review.

\section{2) Radiative forcing induced by volcanic eruptions and uncertainty}

Estimating the anomalous radiative forcing induced by volcanic eruptions is a difficult task, since there are several elements that can affect this budget. Sulphate aerosols are the main drivers of the volcanic impact on the radiative budget, but other elements emitted during the eruption, such as water vapour or carbon dioxide emission can play a role as well, but on longer time scale than the one (decades) considered in this paper. Indeed, although water vapour usually represents the largest amount of ejected gas, it has a very weak climate impact because it is recycled rapidly through rainfall. The amount of carbon dioxide emitted in one eruption is usually not large enough to strongly affect the global atmospheric $\mathrm{CO}_{2}$ concentration, so that it is mainly when accumulated over lots of volcanic eruptions that it can lead to substantial radiative forcing over a long time frame (Robock 2000).

The $1991 \mathrm{Mt}$. Pinatubo eruption is the sole large volcanic eruption that has been well observed, and therefore it is used as a reference for reconstructing the aerosol loading and radiative forcing impact of the other past volcanic eruptions. Observations of sulphate aerosols have been enabled by the instrument Stratospheric Aerosol and Gas Experiment II (SAGE II) which was aboard the Earth Radiation Budget Satellite (ERBS) satellite launched in 1984. It measured sunlight through the limb of the Earth's atmosphere in seven spectral wavelengths (from 0.3851.02 micrometres) and provided data as a function of latitude and altitude (vertical profiles) with unprecedented temporal and spatial resolution (Sato et al. 1993, Figure 2). It had, however, a huge gap in the lower stratosphere in the tropical area, where data were missing.

As explained above, after a tropical explosive volcanic eruption, the formation of sulphate aerosols modifies the optical depth of the atmospheric layer over the whole globe. A primary consequence is an increased planetary albedo due to the aerosol size distribution, which reflects back to space the incoming shortwave solar radiation and thus reduces the net radiative balance at the top of the atmosphere. Yet, this influence depends on the eruption strength and latitude (Timmreck et al. 2009). Some plume models (e.g. Active Tracer High Resolution Atmospheric Model: ATHAM) can account for the plume dynamics complexity (Oberhuber et al. 1998; Herzog et al. 1998; Graf et al. 1999). Given the very small space scale processes that they represent, they have not been formally incorporated in global climate models yet.

So far, AOGCMs have relied on simplified approaches to account for the radiative impact of volcanic eruptions. The most basic one, still used in a few simulations participating to CMIP5, is to consider that volcanic eruptions simply lead to a modulation of the total solar irradiance (TSI) available for the climate system at the top atmospheric level of the model (Timmreck 2012). Nevertheless, such an approximation does not account for the vertical and horizontal structure of the optical depth change associated with volcanic eruptions ( $c f$. Figure 2). Many models (i.e. GISS, NCAR, MIROC, etc.) resolve the three-dimensional propagation of sulphate aerosols, although they usually specify their distribution rather than calculating it. When specified, the optical depth of the relevant atmospheric layer is directly modified in the model, following the latitudinal evolution of the aerosols derived from an offline transport model (e.g. Ammann 2003). This approach not only allows a time-space evolution of the radiative cooling on the Earth surface but also a representation of the absorption of energy by volcanic sulphate aerosols in the stratosphere, leading to an upper-level warming just after the eruption. This upper-level warming and lower-level cooling of the atmosphere was clearly observed after the Mt. Pinatubo eruption (Labitzke \& McCormick 1992).

Reconstruction of the sulphate aerosols burden and propagation for past eruptions has been proposed based on backward trajectory experiments of sulphate concentration recorded both in the Greenland and Antarctic ice cores. A few attempts have been made (Ammann et al. 2007; Gao et al. 2008) and lead to slightly different estimates in the timing and magnitude of volcanic eruptions over the last millennium (Figure 3), depending on the methods and data used. 
Yet, all of them indicate that Samalas eruption, which has only been located very recently (Lavigne et al. 2013), is the largest eruption over the last millennium, and even over the last 2500 years according to a more recent reconstruction going further back in time and still based on sulphate measurements within ice cores (Sigl et al. 2015).

\section{3) Impact on global temperature}

Volcanic eruptions primarily impact the global radiative budget of the Earth, leading to a global temperature drop, as described in the introduction and first noticed by Lamb (1970). For example, the cooling impact of the Mt. Pinatubo eruption is evaluated to be of around $0.5 \mathrm{~K}$ (Soden 2002). Brohan et al. (2012) used an unprecedented collection of observations of logbooks preserved in the British library to estimate the response of the global climate to the 1815 Tambora and the 1809 unknown eruptions. They found a global temperature response to these two eruptions of the same order of magnitude, which can be interpreted as rather modest given the reconstructed IVI of these eruptions. Nevertheless, it remains difficult to assess the exact temperature response to a particular eruption solely from observations given that the forced volcanic signal is superimposed upon natural variability, for example related to ENSO, AMO and NAO among others (Zanchettin et al. 2013a), which can be superimposed on volcanic eruptions' impacts (Lehner et al. 2016). In addition, Canty et al. (2013) argued that taking into account the ocean circulation changes due to the eruptions could lower the estimate of the radiative directly induced global temperature cooling by a factor of two. Nevertheless, their analysis should be considered with caution because they used an AMO index as a proxy of ocean circulation, while the latter includes by construction (spatial average of SST anomalies) the radiative changes due to volcanic eruptions (this effect is further discussed in section 5).

Figure 4 represents the global temperature anomalies after the five largest volcanic eruptions since 1850 (1883 Krakatau, 1902 Santa María, 1963 Mt. Agung, 1982 El Chichón, 1991 Mt. Pinatubo eruptions) in two different datasets. These five events constitute an ensemble of five members, and the ensemble mean is thus interpreted here as an estimate of the forced volcanic signal. This assumes that the internal variability, here considered as noise, has been averaged out. The spread of the response to the individual events may give an idea of the intensity of this noise, although it might also reflect the heterogeneity in the magnitude of the considered volcanic eruptions. A clear cooling occurs in the ensemble mean, peaking between 1 and 2 years after the onset of the volcanic eruption. The global temperature returns back to normal conditions around 5 years after the onset of the eruption, illustrating that due to the inertia of the climate system, and notably the ocean, volcanic impacts on climate can persist even after the radiative forcing from volcanic aerosols has disappeared. This cooling is clearly visible for all the screened individual eruptions, except for the 1982 El Chichón eruption, which was occurring while a big El Niño event was registered the same year.

Within the last millennium context, the sampling of volcanic eruptions is more adequate to produce robust statistics and minimize the pollution of the signal by the natural variability. Nevertheless, the impact of volcanic eruptions on temperature at hemispheric scale is still debated, because important differences persist between paleoclimate data and simulations. Until recently, the signature of very large volcanic eruptions like Samalas or Tambora was not clearly visible in temperature reconstructions of the last millennium, in particular from tree ring data, which is known to capture particularly well interannual climatic variations. To explain this mismatch, Mann et al. (2012) proposed that years with very cold anomalies, similar to the year without a summer following the Tambora eruption in 1816 (Luterbacher \& Pfister 2015), may prevent trees from growing and de facto inhibit the production of any ring. Such an issue would have a very strong impact on the estimation of the age of trees and consequently on the related chronology in the climate reconstructions, since some years may be missing in the account of layers.

Schurer et al. (2014) showed that volcanic eruptions can explain most of the forced variability over the last millennium as compared to solar variations. These findings were 
confirmed over the last two millennia by using continental scale reconstructions by PAGES $2 \mathrm{k}-$ PMIP3 group (2015). Concurrently, climate models seem to produce too strong surface cooling related to volcanic eruptions (Fernández-Donado et al. 2013). An overestimation of Northern Hemisphere temperature response to volcanic eruptions has also been found by Schurer et al. (2013) using detection-attribution analyses.

A recent study (Stoffel et al. 2015) has overcome the issue on tree rings and reconciled discrepancies between observed reconstructions and model results. First, from a new tree-ring compilation based on density of wood instead of classical ring width, they showed that the volcanic-induced cooling is indeed significant in the Northern Hemisphere. Second, they provided evidence that climate models forced with aerosol properties explicitly calculated by an aerosol process model containing a fully explicit high-resolution size-resolving aerosol microphysical module (Bekki et al. 1997), simulate weaker radiative impacts on surface temperature than the previous generation of models. This study, among others, illustrates that the complexity of the forcing and its representation in models are key for assessing the global impact of volcanic eruptions, and confirms previously reported results and hypothesis by Timmreck et al. (2009) and Timmreck (2012).

Furthermore, the cooling induced by volcanic eruption is not spatially uniform and is notably following the sulphate aerosol distribution evolution, which is varying in space and time (Figure 2). Figure 5 shows an example of the spatial response from the CNRM-CM3 climate model. Changes in aerosol optical depth from Ammann et al. (2007) reconstruction of volcanic eruptions are prescribed in this model version that accounts for the vertical-latitudinal variations of the aerosols as depicted in Figure 2. Figure 5 shows the near-surface temperature composite, as simulated in a last millennium experiment with the CNRM-CM3 model (Swingedouw et al. 2011) in response to the 19 volcanic eruptions (cf. Annex Table 1) of amplitude larger or equal to1991 Mt. Pinatubo eruption in terms of global radiative forcing from Ammann et al. (2007). The zonally averaged temperature response at the surface during the months where sulphate aerosols strongly influence the radiative budget (1 to 18 months, typically depending on the eruption, see Annex Table 1), mainly follows the forcing (Figure 5 right panel), with stronger changes in the tropical area than in the high latitudes (Figure 5, see also Mignot et al. (2011) for an example with another climate model). Despite large-scale overall cooling, slight warming occurs locally in the Central Pacific or in Northern Europe for instance. These two features may be indicative of a dynamical response of the climate system, which could modulate or even mask the direct radiative forcing signal. This is explored in the following.

\section{4) Impact on the NAO}

\section{Early observations}

Using Russian meteorological data, Groisman (1985) noticed that the signature of the 10 largest eruptions since 1815 on Siberian winter temperature is a warming rather than a cooling. The pioneering work of Robock \& Mao (1992) related this regional anomaly to NAO and AO variations in response to volcanic eruptions, thus inspired by the very strong positive phase of the NAO observed in winters 1992 and 1993 following the Mt. Pinatubo eruption. The NAO+ phase in winter leads indeed to enhanced westerlies, advecting relatively warm air from the ocean into the Eurasian continent. Such a link has been later on reported for the whole instrumental era (Christiansen 2008; Driscoll et al. 2012). Nevertheless, the limited number of volcanic eruptions considered (less than 10) casts doubt on the significance of such results. Moreover, when looking at the response to the 5 largest eruptions over the instrumental era, the composite $\mathrm{NAO}+$ signal during the year of the eruption is very weak (Figure 6) and not significant at the $90 \%$ level. This response remains insignificant during the few years following the eruption. It can be related to the intrinsic very chaotic nature of the NAO, which leads to large internal variations that mask the forced signal (weak signal to noise ratio). 
To increase the sampling size and magnitude of the considered eruptions, similar analyses have been extended over the last millennium. Because sea level pressure reconstructions are not directly available, Fischer et al. (2007) used a reconstruction of the European temperature over the last 500 years (Luterbacher 2004) to show that the winters following the 15 largest eruptions are associated with strong warming of northern Europe, which could be interpreted as a fingerprint of a positive phase of the NAO (Casado et al. 2013). Zanchettin et al. (2013b) even suggested that this impact on NAO could last up to almost a decade for the 9 strongest volcanic eruptions of the last 500 years. Nevertheless, this result (Zanchettin et al. 2013b) was again based on fingerprints of the NAO only from Europe and comprised a limited selection of volcanic eruptions due to its short time span. Recently, a new multi-proxy reconstruction of the NAO covering the last millennium, based on several proxies located over the whole North Atlantic sector and using a robust statistical methodology showed that the NAO is almost systematically positive two years after 11 major volcanic eruptions of the last millennium (for details on this result see Ortega et al. 2015). We reproduce this result in Figure 7a for a narrower selection of 8 eruptions for which the year of the occurrence is better constrained ( $c f$. Table 2). This refined composite shows that the maximum NAO response still occurs two years after the eruption (i.e. lag 1), but that significant signals (at the $90 \%$ confidence level) develop from the first to the fourth year. There is also a significant ( $90 \%$ confidence level) positive NAO phase two years before the eruption, which cannot be possibly caused by volcanic aerosols. This suggests that some dating uncertainties, either in the reconstruction or in the occurrence of the eruptions, are still present. This problem is not specific to the NAO, as it is one of the key issues in analyses with proxy-based reconstructions, especially when looking at the impacts on specific years.

\section{Processes leading to the NAO response}

The impact of volcanic eruptions on the low-level atmospheric circulation is here illustrated again with the last millennium simulation from the CNRM-CM3 model (Swingedouw et al. 2011). The composite for the sea-level pressure field over the 19 largest eruptions of the last millennium (Annex Table 1) clearly shows a positive A0-like structure, with strong negative anomalies over the North Pole (Figure 8). There is a similar signal in the Southern Hemisphere, which may indicate a concomitant strengthening of the Southern Annular Mode. Figure 9b represents the temporal response of the SLP north of $65^{\circ} \mathrm{N}$ for the different eruptions considered in Figure 8, in order to give information on the diversity of responses following the individual eruptions. Consistently, very negative SLP values are found north of $65^{\circ} \mathrm{N}$ for the first and the second winters after each of the considered eruptions (Figure 9b), a signal that dissipates afterwards. Nevertheless, this representation of the NAO response to volcanic eruptions in CNRM-CM3 is an outlier among the PMIP3 (Braconnot et al. 2012) model database. Indeed, Figure 7b shows that most of the PMIP3 models (Table 3), but CNRM-CM3 and CCSM4, do not produce a statistically significant (above 95\% level) positive NAO response after the largest volcanic eruptions of the last millennium, contrarily to the recent multi-proxy reconstruction of the NAO (Figure 7a). This lack of NAO response in most of the climate models has also been shown in Driscoll et al. (2012) for the major volcanic eruptions over the instrumental era (1850-2005).

Most of the mechanisms proposed to explain a possible impact of volcanic eruptions on the NAO involve the stratosphere and the polar vortex. The radiative impact of the volcanic eruption leads to a warming of the lower tropical stratosphere, which may enhance the meridional temperature gradient between the low latitudes and the poles in the lower stratosphere. Through thermal wind balance, such a modification induces in turn an enhancement of the zonal wind in the mid to polar latitudes in the stratosphere, hence strengthening the polar vortex. A propagation through downward reflection of stationary waves (Kodera 1994; Shindell et al. 2003) towards the troposphere can then modulate the sea level pressure and hence the phase of the NAO (Robock 2000). Nevertheless, the mean state of the meridional temperature gradient in the stratosphere is inversed as compared to that in the troposphere (warm in the tropics and cold in the poles), which is therefore questioning the 
validity of the former explanation. Furthermore, other processes may play a role in driving the polar vortex changes following the eruptions, such as changes in ozone or upward propagating waves from the troposphere (Stenchikov et al. 2006; Toohey et al. 2014). However, their exact role is still not totally clear. Assuming that the volcanic eruptions-NAO+ relationship is true (Figure 7a), deficiencies of the last generation of global climate models to reproduce it may be notably related to a coarse representation of the stratosphere in the last generation of global climate models, or a simplistic representation of the associated radiative forcing (Graf et al. 2014). The reasons for the different behaviour of CNRM-CM3 and CCSM4 with respect to the other models remain to be elucidated.

A recent study (Barnes et al. 2016) has highlighted that the 1991 Mt. Pinatubo eruption triggered robust changes of both stratospheric and tropospheric circulation in the CMIP5 models, but these changes do not project exactly on the NAO. Bittner et al. (2016) confirmed that CMIP5 models do show a polar vortex strengthening in response to Pinatubo or Krakatau-like volcanic eruptions, which nevertheless do not propagate to the surface ( $c f$. their Figure 4). Using a very large ensemble of simulations performed with the MPI-ESM model, Bittner et al. (2016) also showed the very weak signal-to-noise ratio for the wind speed response to volcanic eruptions, with more than 10 members necessary to isolate a response for large eruptions like Pinatubo or Krakatau.

\section{Potential implications for climate prediction}

The identification of possible volcanic impacts on the NAO has great implications for seasonal prediction. To illustrate and evaluate the potential skill associated to the historical volcanic eruptions, we analyse retrospective predictions, called hindcasts, using a specific decadal forecast system (Meehl et al. 2014) based on the EC-Earth atmospheric-ocean general circulation model (Hazeleger et al. 2012). Initial atmospheric and land-surface conditions for the predictions are derived from a physical interpolation of ERA-Interim data, while the version 2.1 of GLORYS reanalysis (Ferry et al. 2010) is used to initialize the ocean and sea-ice components. Ensemble members are generated using singular vector perturbations in the atmosphere. The full protocol used to perform these hindcasts is described in Batté \& Doblas-Reyes (2014). The EC-Earth model was not included in the previous PMIP3 database since no last millennium simulation was available. The volcanic forcing is based on the GISS observational dataset, described in Sato et al. (1993) and updated up to 2012 by M. Sato (http://data.giss.nasa.gov/modelforce/strataer). It is resolved in 24 different latitudinal bands, and expressed in terms of Aerosol Optical Depth (AOD).

In the following, we consider 5-member ensembles of hindcasts initialised in November of the years of the eruptions Mt. Agung (March 1963), El Chichón (April 1982) and Mt. Pinatubo (June 1991) and subsequently integrated with and without applying the effects of volcanic eruptions. On average, the surface temperature response is comparable to what has been found in previous studies, with a significant cooling over the tropics and a noisier signal at mid to high latitudes, resulting from the superposition of the direct volcanic cooling, the dynamical response and large-scale internal variability. In these experiments, we did not find any consistent positive NAO signal during the first two winters following the eruptions, but a significant positive NAO signal seems to emerge on average in the third winter (Figure 10a). It is solely related to El Chichón as it becomes evident when considering the eruptions individually (Figure 10b). Indeed, no positive NAO signal emerges after the Mt. Pinatubo and the Agung eruptions, neither for the first two winters, nor for the third one. Incidentally, the observations do not support the positive NAO during the third winter following the 1982 El Chichón eruption, and rather show a negative one as for 1963 Agung eruption (Figure 10b).

We argue here that the internal variability of the NAO overwhelms the volcanic signal for medium-sized eruptions, such as Pinatubo, Agung or El Chichón (cf. Bittner et al. 2016). This is in agreement with Toohey et al. (2014) who found Pinatubo-like eruptions to be too small to affect directly the polar vortex. Consequently, it is difficult to detect this signal, if any, with a limited ensemble of forecast runs (here 5 members). Finally, the skill of the current seasonal forecast systems to predict the NAO is generally very low, and often not significant. We show 
here that this conclusion is also valid during periods with Pinatubo-like eruptions. It remains then possible that the positive NAO response observed during the two winters following El Chichón and Pinatubo eruptions has occurred simply by chance and is thus due to internal variability.

In addition to the considerable weight of internal variability that makes it difficult to predict the dynamical response to volcanic eruptions, many studies highlighted that climate conditions, such as ENSO and the quasi-biennial oscillation (Thomas et al., 2009), can also modulate this response (Zanchettin et al. 2013a). The AMO is also likely to impact the atmospheric response to volcanic eruptions in the North Hemisphere: the increase of Arctic sea-ice concentration during a cold state of the AMO may induce feedbacks that favour the positive phases of the NAO the third winter after the eruption onset (Ménégoz, personal communication).

To conclude, some observational evidence exists for a positive NAO response to volcanic eruptions as well as a few physically consistent mechanisms. However, the weak signal-to-noise ratio associated to mid-sized (Pinatubo-like) eruptions, and the fact that the exact mechanisms at play are still not well understood and captured in CMIP5 models, limits the prospects of predictability of such atmospheric dynamical responses.

Volcanic eruptions could also impact the atmospheric circulation in the Southern Hemisphere. Marshall (2003) observed strong positive phases of the Southern Annular Mode the years following the Agung eruption. The link between volcanic eruptions and the polar vortex position has been widely investigated in the Northern Hemisphere. Theoretically, this link also exists in the Southern Hemisphere, as illustrated in Figure 6. Nevertheless, the exact dynamical mechanism in the Southern Hemisphere has not been analysed in detail yet.

\section{5) Impact on ENSO}

\section{Observational evidence}

The variability in the Pacific Ocean can also be affected by volcanic eruptions. For four out of five of the largest eruptions over the industrial era, a warming signal is found in the tropical Pacific Niño 3.4 region during the same year as the eruption (Figure 11). Nevertheless, as highlighted by the exact starting date of the eruption in Figure 11b, in most of the cases a warming was already happening before the onset of the eruption, suggesting that an El Niño event was developing before the eruption begins. Observing the early stage of El Niño event at the beginning of the year does not necessarily mean that it will develop, the year 2014 being a nice example of this, with potential for an El Niño event from knowledge of the first half of 2014 (World Meteorological Organisation 2014), but no clear development of the event afterwards. For the Santa María eruption (1902), the El Niño event already occurred before the eruption onset, so that only Mt. Pinatubo and El Chichón can be considered to be followed really by a large El Niño event even though there were already hints of an early stage El Niño before the eruption.

An El Niño response is consistent with the cooling gap simulated in the central tropical Pacific in the CNRM-CM3 model after the 19 largest eruptions from last millennium (Figure 5). This suggests a possible volcanic contribution since this response is opposite to the expected direct radiative cooling impact as documented in Tung \& Zhou (2010). In their analysis they performed linear regressions on the HadISST (Rayner et al. 2003) and the Extended Reconstructed SST (ERSST, Smith et al. 2008) data sets to remove the direct volcanic radiative cooling signature on SST, using the estimates of aerosol optical depth from Sato et al. (1993). From this procedure, they found a large positive ENSO-like pattern from both SST datasets in response to major volcanic eruptions a year after. Maher et al. (2015) recently confirmed this result using sea level height data as a proxy of ENSO activity. Nevertheless, given that El Niño events occur naturally every 2 to 7 years, the probability of having an El Niño by chance just after a volcanic eruption is clearly high, due to the short return period. 
Similarly to the NAO, few analyses have tried to evaluate if El Niño events have been excited by volcanic eruptions over the last millennium. Adams et al. (2003) went back to 1649 using two proxy-based ENSO reconstructions and found a doubling of the chance of having an ENSO positive phase after a selection of 13 major volcanic eruptions over the period 1649-1979. This result has been confirmed in other long reconstructions (McGregor et al. 2010; Wilson et al. 2010; Li et al. 2011, cf. Figure 12a). These studies based on paleoreconstructions over the last millennium therefore corroborate the fact that volcanic forcing exerts a discernible influence on ENSO. In terms of impact, Chikamoto et al. (2016) noticed that, for the climate models that indicated such an impact of volcanic eruption on ENSO, a large effect on the primary productivity of the Tropical Ocean for a few years is also observed, due to modifications of nutrient supplies (Seferian et al. 2014).

\section{Mechanisms}

According to the thermostat mechanism described in Clement et al. (1996), strong tropical eruptions induce, in the Zebiak \& Cane (1987) ENSO conceptual model, a surface cooling which reduces the temperature contrast between the surface ocean and the underlying thermocline in the Eastern equatorial Pacific. This configuration reduces the westward climatological SST gradient, which may trigger the chain of events (notably the so-called Bjerknes feedback) that favours El Niño development the following year (Seager et al. 1988; Emile-Geay et al. 2008).

Despite observational evidence, AOGCMs have difficulties to simulate such a response. This is illustrated in Figure 12b for the PMIP3 simulations, which show very few El Niño events in the two years following the 8 largest eruptions of the last millennium. We focus on 8 eruptions to be consistent with the sample size in Table 2, including 8 large eruptions in three different reconstructions of volcanic forcing, all of them with precise dating from historical records. These are not necessarily the same eruptions analysed in the different PMIP3 simulations, as these use different forcing records (and the 8 largest eruption vary among them). The only models that show significant warming in Niño3.4 box are GISS-E2-R, IPSL-CM5A-LR and CNRM-CM3. This suggests that the direct response of the ocean dynamics to the radiative forcing proposed in Clement et al. (1996) may not be the dominant mechanism in all the models. As for the NAO, the proposed theoretical mechanism of response to volcanic eruptions (thermal wind balance for the NAO, thermostat for ENSO) is undetectable due to the presence of other processes and climate noise. In MIROC AOGCM, Ohba et al. (2013) found that the uniform reduction of incoming surface solar radiation in winter can, depending on the ENSO phase when the forcing is maximum, either initiate or amplify an El Niño event, or shorten the duration of La Niña events. This points out the sensitivity of the tropical Pacific response to the month of the onset of the radiative perturbation, and therefore of the precise timing of the volcanic eruptions, as well as initial conditions. Opposite fingerprints can be even found in some models. In CCSM3 and MPI-ESM models, it has been shown that in response to the tropical reduction of incoming surface solar radiation, changes in wind pattern related to tropical cooling can initially lead to a strong negative phase of ENSO (e.g., McGregor \& Timmermann 2011; Zhang et al. 2013). Strong volcanic eruptions of different magnitude and seasonality implemented in the CCSM3 model induce indeed enhanced trade winds in the eastern equatorial Pacific, which lead to a deeper thermocline in the West and warmer SSTs after a period of several months. The build-up of the warm water volume in the equatorial Pacific, will eventually favour the growth of SSTs (within the framework of the recharge-discharge oscillator, $c f$. Jin 1997) during the ensuing El Niño.

Thus, the specific details of the short-term dynamical climatic responses to volcanic eruptions remain unclear, as differences in experimental protocols and biases in model representations of ENSO variability (Guilyardi 2006) could affect their response to volcanic forcing. The analysis of cloud radiative feedbacks in convection/subsidence dynamical regimes in the CMIP3 models (Bony \& Dufresne 2005) shows that important uncertainties among AOGCMs are related to the simulation of marine boundary layer clouds. The sensitivity of the ENSO response to volcanic forcing could also reflect such a model bias. 
Thus, an in-depth intercomparison of the feedback response to a standardized volcanic event (same magnitude, starting date, number of members) in different models would be useful to determine precisely the dynamical differences for the ENSO response to volcanic eruptions in climate models. This would represent a very useful test bed to evaluate the dynamical response of the models to volcanic forcing and thus to validate them as well as the processes at play.

\section{6) Impact on the AMO and AMOC}

\section{Volcanic signature on the AMO}

The AMO is classically viewed as the surface fingerprint of the modulations of the large-scale Atlantic Meridional Overturning Circulation (AMOC), which transports large amounts of heat northward in the Atlantic (Figure 1). This view mainly arises from model studies. In so-called control simulations, where the external radiative forcing is kept constant, positive AMO phase typically lags AMOC intensification by 5 to 10 years (e.g. Knight et al. 2005; Persechino et al. 2013; Ruprich-Robert \& Cassou 2014). Some recent observational hints (McCarthy et al. 2015) partly support this link with the ocean circulation, although they are not based on direct observations of the AMOC. As discussed above, several definitions exist in literature for the AMO. They are all based on an SST index, typically computed from SST anomalies averaged over the North Atlantic $\left(0-60^{\circ} \mathrm{N}, e . g\right.$. Enfield et al. 2001). This definition implies that the AMO includes the direct radiative influence of the external forcing (greenhouse gases, anthropogenic aerosols, volcanic eruptions...). Several studies indeed suggest that AMO variations over the instrumental era have been modulated directly, i.e. through radiative changes, by the external forcing (Otterå et al. 2010; Knudsen et al. 2014; Booth et al. 2012) and indirectly through the effect of the AMOC for example (Marini et al. 2013). Among these forcings, volcanic eruptions have a large imprint on the SST and therefore a great potential to influence the AMO variability.

Figure 13 illustrates this effect of volcanic eruption, by using two simulations of the last millennium performed with the IPSL-CM4 climate model, one forced with the sole variations of the total solar irradiance (Servonnat et al. 2010), and the other one where the effect of the volcanic eruptions is added to variations of the solar irradiance (Mignot et al. 2011). The correlation between the modelled AMO and the AMO reconstruction from Gray et al. (2004) is of 0.18 (not significant at the $90 \%$ level) when only solar variations are accounted for; it reaches 0.45 (significant at the $95 \%$ level) when volcanic eruptions are also included as a forcing. This shows the crucial role played by volcanic eruptions for capturing the reconstructed variations of the AMO as defined above. This has also been illustrated by Zanchettin et al. (2012) and Lohmann et al. (2015). More precisely, Figure 14 shows a clear reduction of the AMO index following the 8 major volcanic eruptions of the last millennium in the PMIP3 simulations as well as in a few reconstructions of the AMO (and some local SST proxies in the North Atlantic) over the last millennium. This reduction is related to the surface cooling induced by radiative impact of the volcanic eruptions as described above.

The definition per se of the AMO index is crucial to assess the Atlantic response to the volcanic eruptions. When the term AMO strictly refers to the internal variability part of the climate system over the North Atlantic, as opposed to externally-driven SST changes, a new computation of the index is required. Over the instrumental era, a gradual warming due to greenhouse gases is superimposed on internal SST variability. To isolate the internal part, it has thus been proposed to subtract, for each time step, the anomalous global mean SST from the North Atlantic average (Trenberth \& Shea 2006). Detrending linearly or through regression on $\mathrm{CO}_{2}$ concentration time series has also been proposed. All these definitions account differently for the response of external forcing, including or not volcanic forcing. Sensitivity to the definition can be compared within models (Figure 14b and Figure 15a). Removing the global signal (Figure 15a) leads to a much weaker impact of volcanic forcing (compare with Figure 14b), suggesting a minor role of dynamical adjustments by the ocean circulation, as compared to the direct effect of the radiative forcing on North Atlantic temperatures. 


\section{Impact on the AMOC}

In spite of its crucial climatic relevance, there is no robust proxy for the AMOC variations over the last 1000 years, and very limited observations over the modern period (roughly a decade, at specific latitudes). Climate models are thus the primary tool to investigate the response of the AMOC to volcanic eruptions. As described above, volcanic eruptions may induce a rapid reorganization of the atmospheric circulation. In that case, it provokes a rapid barotropic response of the oceanic circulation, which has imprints on its zonally-averaged stream function, the latter being a good representation of the AMOC. In the latitude-depth plane, a weak cyclonic cell appears at high latitudes and an anticyclonic anomalous circulation in the subtropics, both inducing anomalous downwelling at mid-latitudes (Mignot et al., 2011; Zanchettin et al., 2011). This particular structure is forced by an anomalous wind-stress curl at the surface that is dynamically consistent with positive NAO-like conditions.

This fast-developing anomalous circulation is significant but not relevant in terms of anomalous heat transport and long-term climatic effects. At decadal timescales, on the other hand, most model studies show a large-scale intensification of the AMOC 5 to 15 years after the volcanic eruptions (Stenchikov et al. 2009; Zanchettin et al. 2011; Mignot et al. 2011; Ortega et al. 2011; Ding et al. 2014; Swingedouw et al. 2015), which may imply more substantial redistribution of the heat received in the ocean. Yet, mechanisms differ among studies: Stenchikov et al. (2009) and Ortega et al. (2011) invoked a thermohaline mechanism after the cooling of the ocean induced by the direct radiative forcing of the volcanic eruption, while Zanchettin et al. (2011) and Mignot et al. (2011) found a delayed response to atmospheric perturbations. Other studies propose other mechanisms. For instance, using the HadCM3 climate model, Iwi et al. (2012) reported an AMOC intensification after a Krakatau-like eruption through the runoff reduction towards the Arctic basin, but they also found that this effect was not seen after a weaker Pinatubo-like eruption, thereby illustrating a strong sensitivity to the magnitude of the eruption. Using the IPSLCM4 model, Mignot et al. (2011) also found a very specific response for the AMOC after the intense and cumulative eruptions that occurred around 1250 and 1300, where profound and long-lasting effects on the sea ice extension (e.g. Sicre et al. 2013 for a proxy evidence) capped oceanic deep convection in the subarctic Seas and favoured an AMOC decrease, consistently with Zhong et al. (2011). Miller et al. (2012) even proposed that this AMOC weakening in response to the very large 1257 Samalas eruption could be irreversible, as found in CCSM3 simulation, which may have participated to the onset of the little ice age according to these authors.

The importance of the amplitude and phasing of the volcanic eruptions for the AMOC response has been further highlighted by Swingedouw et al. (2015, their Figure S11) who showed that depending on the magnitude of the eruption, different areas of the northern North Atlantic may be affected, thereby possibly leading to opposite anomalies of the oceanic deep convection. The latter study highlights the crucial interplay between the AMOC internal variability and the volcanic eruptions. Indeed, moderate volcanic eruptions similar to $1991 \mathrm{Mt}$. Pinatubo eruption were shown to impact specifically the Nordic Seas, partly via a sea-ice feedback, which had been shown to be a key region for the internal variability of the AMOC in the IPSL-CM5A-LR model (Escudier et al. 2013).

In the four PMIP3 models for which the AMOC diagnostics were available, three of them show a fast AMOC response in the first two years following the 8 largest eruptions ( $c f$. Table 2) of the last millennium (Figure 15b), which may be explained by a rapid dynamical (Mignot et al. 2011) or thermodynamical (Ortega et al. 2011; Stenchikov et al. 2009) adjustment to the atmospheric response to the eruption. CNRM-CM3 is the one showing the largest response in the first few years both for the AMOC and the NAO (Figure 9), giving credit to the link between atmospheric and AMOC responses. On longer timescales, we find a second response from years 10 to 20 in 3 out of 4 of the available models. Such a response is in agreement with Swingedouw et al. (2015) results based on weaker eruptions. Nevertheless, IPSL-CM5A-LR model shows a shorter delayed AMOC maximum in response to the weak eruptions (Pinatubo-like) studied in Swingedouw et al (2015) than for the 8 largest eruptions of the last millennium studied herein 
(20 years for the latter instead of 10-15 years for the former). This may be linked to the different SST and sea ice response discussed above (cf. Figure S11 in Swingedouw et al. 2015) and probably requires dedicated sensitivity experiments.

These changes in the AMOC can then lead to a modification of the AMO, and support the role of volcanic eruptions as a metronome for AMO variability. To illustrate the time evolution of the AMO (here just defined as averaged SST over the North Atlantic) and AMOC relationship, we compute the 100-year sliding correlations between these two elements of the climate variability in the same four last millennium PMIP3 simulations (Figure 16). In the control simulations, such correlation is almost stationary and maximum when the AMO lags the AMOC by 5 to 10 years, depending on models (not shown). This dynamical link between AMOC and AMO still appears in Figure 16 but during the periods of major volcanic activity, the link between AMOC and AMO is particularly strong in the three models that do show a slow response of the AMOC to volcanic eruptions (CCSM4, IPSL-CM5A-LR and MPI-ESM), including at short time lags or even in phase. We attribute this strengthened link to an enhanced variability of the AMOC in response to volcanic eruptions, which is then influencing the AMO later on. In IPSL-CM5A-LR, significant correlation is even detected when the AMO leads the AMOC. This is attributed to the fact that eruptions influence the AMO in phase, through a global cooling, and then by the AMOC with a delay, as discussed above, so that the two show coherent variations induced by the same external factor. The fact that this effect is not seen in other models may be due to specific AMOC sensitivity in these models and remains to be clarified.

Finally, the simulated responses to the forcing can be sensitive to the state of the climate system, and of the ocean in particular, at the time of the eruption. This has been shown in particular by Zanchettin et al. (2013a) in the MPI-ESM model and related to sea-ice interactions, which could be sensitive to the initial state.

\section{7) Conclusions}

We have reviewed the impact of large tropical explosive eruptions on three key climatic modes of the Earth. In addition to a general discussion of the previous literature, novel diagnostics have been shown, based on instrumental observations, last millennium reconstructions and model simulations, including decadal forecasts, to illustrate and evaluate the state-of-the-art level of knowledge and understanding on this topic.

The basic climatic response to volcanic eruptions is a global cooling of the Earth's surface, associated with a warming of the lower stratosphere. Yet, we have shown that even the assessment of this primary response needs a careful use of proxy data and elaborated model analyses.

We have shown that the impact of volcanic eruptions on the NAO is still not entirely clear from direct observations, because in the presence of climate noise, it may require eruptions larger than $1991 \mathrm{Mt}$. Pinatubo eruption to excite a dynamical response. Longer time series using proxy data do show a robust positive NAO response in the four years following the major eruptions of the last millennium. The climate models analysed here do not all reproduce such a response, and the reasons for their discrepancies are not yet understood. Making progress on this issue will require further dedicated model experiments and analyses of the atmospheric dynamical responses, with models including a more elaborated representation of the coupling between the stratospheric chemistry and the dynamics (e.g. LeGrande et al. 2016). The possibility for seasonal to multi-annual prediction of the NAO following a Pinatubo-like eruption has been evaluated from one single climate forecast system. No firm conclusions can be drawn and a multi-model assessment is required to evaluate more robustly the impact of the volcanic forcing in a predictive context for variables beyond global mean temperature.

Concerning ENSO, we do find a significant positive ENSO phase following the volcanic eruptions both in historical and last millennium reconstructions. However, such a response is again generally not found in climate models, with less than half of them being able to reproduce 
it. This lack of response in several models is suspected to arise from well-known model deficiencies and biases to simulate ENSO variability and the underlying mechanisms.

The impact of the eruptions on the AMO has been also analysed. A negative AMO anomaly has been found in the first few years following the eruptions both in models and reconstructions. This signature, however, is not specific to the North Atlantic and it is of the same amplitude as for the global mean SST. This suggests that it emerges from the direct radiative impact of volcanic eruptions, leading to a global cooling of the surface ocean. This is an important issue, since the AMO is mainly affecting climate through its impact on meridional gradient of temperature, related with AMOC heat transport. It is thus rather an AMO definition where global radiative signal is removed ( $c f$. Trenberth \& Shea 2006) that should be considered in order to consider climate consequences. A longer multi-decadal response of the AMO, defined without global mean temperature, has been found in some models. Such a response could be related with the long term AMOC adjustment after the volcanic eruptions. Nevertheless, the exact timing and duration is quite variable, which might be due to the facts that internal AMOC variability is highly model-dependent (Menary et al. 2015) and the AMOC response to volcanic eruptions is still poorly constrained.

In view of these different results, the hypothesis of volcanic eruptions playing the role of a pacemaker for the internal climate variability is partly supported. If confirmed, such an effect may help to better estimate seasonal to decadal predictability of the climate system, but before that, the major discrepancies in its representation among the different climate models need to be resolved.

In general, the responses described by models and observations are not robust. The magnitude of the eruption required for achieving a significant signal-to-noise ratio as well as the importance of its exact timing are not well understood yet. Hardly any studies propose a systematic investigation of these issues. Furthermore, a number of key elements can improve the representation of the volcanic eruption impacts on climate models, our major tool to understand the processes at play. First of all, the representation of atmosphere microphysics is the key to correctly represent the radiative impact of volcanic eruptions (Stoffel et al. 2015) and therefore the potential three-dimensional temporal changes of the temperature gradient in the atmosphere, and the associated dynamical adjustment. Unfortunately, the current computational cost of such models remains an issue for simulating long time frame like the last millennium. Furthermore, inter-model comparison as well as a better estimate of the importance of initial conditions should be more systematically assessed in the different studies. For this purpose, considering a large number of members in response to well-constrained eruptions is crucial. Only such a coordinated experimental design would allow making progress in our understanding of the differences between climate models in terms of process representation. The VolMIP (Model Intercomparison Project on the climatic response to Volcanic forcing, Zanchettin et al. 2016) project is planning to perform such an inter-comparison in the forthcoming CMIP6 exercise, and will be an ideal test-bed to evaluate model performance and differences. Furthermore, the Decadal Climate Prediction Project (DCPP), another part of CMIP6, is planning to perform multi-model hindcasts including or not the recent volcanic eruptions, and will thus provide an additional test-bed to evaluate climate predictability associated with volcanic eruptions.

Given the small sampling of large volcanic eruptions over the instrumental era, evidences from the paleo-data are crucial to correctly estimate the signal-to-noise ratio induced by volcanic eruptions in the real system. Some recent efforts to improve the robustness of a proxybased NAO reconstruction using models (Ortega et al. 2015) or to constrain the exact timing of main eruptions and their occurrence on a longer time frame (Sigl et al. 2015) are promising approaches. Reconstructions of ENSO, AMO and AMOC are nevertheless still poorly constrained. Efforts to extend indices of all the main climatic modes over the last 2000 years, with an annual resolution in time should be encouraged. This would contribute to further improve our sampling of the volcanic eruptions impact, by classifying them according to their radiative forcing. Such a framework would allow making real improvements in our understanding of volcanic eruptions on climatic modes, as a function of the eruption magnitude and of the initial states of the climate. 
In the present paper, we have only analysed tropical eruptions that occurred during the last millennium, which had comparatively limited intensity. Massive eruptions like Toba in Indonesia (73,000 years BP, VEI of 8) may be helpful to distinguish clear influences on climate variability coming on top of the strong global cooling. Jones et al. (2005) showed, in a climate model simulation, that Toba's eruption led 10 years after to more than a doubling in the AMOC strength. Large eruptions from volcanoes at high latitudes, like the Laki's explosive and then effusive eruption which took place in Iceland in 1783 (122 $\mathrm{Tg}$ of $\mathrm{SO}_{2}, c f$. Highwood \& Stevenson 2003) have also been excluded from this review. A recent model analysis (Pausata et al. 2015) using the NorESM1-M model has shown that such an eruption can also impact ENSO, pushing this mode into a positive phase, and enhance the AMOC in the first decade, before a decrease around three decades after the volcanic eruption onset. In both the previous examples, the possibility of this response to be model dependant can however not be excluded. A proper assessment of such an impact requires again a multi-model analysis, and a comparison with multiple proxies sources, in order to evaluate the robustness of the response proposed by Pausata et al. (2015) for example.

Finally, relatively weak eruptions have recently been argued to participate to the so-called hiatus, a period of smaller increase in temperature between around 1998 to 2012 (Santer et al. 2014). The new observational tools made available by new satellites (notably SAGE II and CALIPSO, $c f$. Vernier et al. 2011) now allow a correct account of the numerous small eruptions on optical depth ( $c f$. Figure 2 from Santer et al. 2014), which were inaccessible until now. Next desirable steps will be to integrate the four-dimensional evolution of aerosols clouds in the climate models, accounting for longitudinal variations as well. Indeed, such a complex forcing pattern can largely modify the large-scale temperature gradient, with potential impacts on the main variability modes. Skillful forecasts of this cloud propagation following medium to large volcanic eruptions will potentially provide an interesting additional source of climate predictability.

Acknowledgment: This research was partly funded by the ANR MORDICUS project (ANR-13SENV-0002-02). It is also funded by the SPECS project funded by the European Commission's Seventh Framework Research Programme under the grant agreement 308378 and by the EMBRACE project with Research Number 282672. To analyse the CMIP5 data, this study benefited from the IPSL Prodiguer-Ciclad facility, which is supported by CNRS, UPMC, Labex LIPSL, which is funded by the ANR (Grant \#ANR-10-LABX-0018) and by the European FP7 ISENES2 project (Grant\#312979). The research leading to these results has received funding from the Ministerio de Economía y Competitividad (MINECO) as part of the VOLCADEC project CGL2015-70177-R. P.O work was supported by the NERC Project "Dynamics and Predictability of the Atlantic Meridional Overturning and Climate Project" (DYNAMOC, NE/ M005127/1). We also thank Patrick Brockmann for help with the figure design and Eric Guilyardi for useful insights on the section dealing with ENSO. Finally, we acknowledge the comments from two reviewers that helped to clarify our arguments and complete the paper with some useful references.

\section{References}

Adams, B.J., Mann, M.E. \& Ammann, C.M., 2003. Proxy evidence for an El Niño-like response to volcanic forcing. Nature, 426(6964), pp.274-278.

Ammann, C.M., 2003. A monthly and latitudinally varying volcanic forcing dataset in simulations of 20th century climate. Geophysical Research Letters, 30(12), p.1657. Available at: http://doi.wiley.com/10.1029/2003GL016875.

Ammann, C.M. et al., 2007. Solar influence on climate during the past millennium: results from transient simulations with the NCAR Climate System Model. Proceedings of the National Academy of Sciences of the United States of America, 104(10), pp.3713-8. Available at: http://www.pnas.org/content/104/10/3713.abstract.

Barnes, E.A., Solomon, S. \& Polvani, L.M., 2016. Robust wind and precipitation responses to the Mount Pinatubo eruption, as simulated in the CMIP5 models. Journal of Climate, in press.

Batté, L. \& Doblas-reyes, F.J., 2015. Accounting for model uncertainty in EC-Earth3 : impact of SPPT on 
seasonal forecast quality. Climate Dynamics, 16, p.3532. Available at: http://dx.doi.org/10.1007/s00382015-2548-7.

Bittner, M. et al., 2016. Using a large ensemble of simulations to assess the Northern Hemisphere stratospheric dynamical response to tropical volcanic eruptions and its uncertainty. Geophysical Research Letters, pp.9324-9332. Available at: http://doi.wiley.com/10.1002/2016GL070587.

Bjerknes, J., 1969. Atmospheric teleconnections from the equatorial Pacific. Journal of Climate, 97, pp.163-172.

Bony, S. \& Dufresne, J.L., 2005. Marine boundary layer clouds at the heart of tropical cloud feedback uncertainties in climate models. Geophysical Research Letters, 32(20), pp.1-4.

Booth, B.B.B. et al., 2012. Aerosols implicated as a prime driver of twentieth-century North Atlantic climate variability. Nature, 484(7393), pp.228-32.

Braconnot, P. et al., 2012. Evaluation of climate models using palaeoclimatic data. Nature Climate Change, 2(6), pp.417-424. Available at: http://dx.doi.org/10.1038/nclimate1456.

Brohan, P. et al., 2012. Constraining the temperature history of the past millennium using early instrumental observations. Climate of the Past, 8(5), pp.1551-1563.

Canty, T. et al., 2013. An empirical model of global climate - Part 1: A critical evaluation of volcanic cooling. Atmospheric Chemistry and Physics, 13(8), pp.3997-4031. Available at: http://www.atmos-chemphys.net/13/3997/2013/.

Casado, M. et al., 2013. Impact of precipitation intermittency on NAO-temperature signals in proxy records. Climate of the Past, 9(2), pp.871-886.

Chikamoto, M.O. et al., 2016. Volcanic eruptions boost tropical Pacific biological productivity. Geophysical Research Letters, in press, p.n/a-n/a. Available at: http://doi.wiley.com/10.1002/2015GL067359.

Christiansen, B., 2008. Volcanic eruptions, large-scale modes in the Northern Hemisphere, and the El Ni??oSouthern Oscillation. Journal of Climate, 21(5), pp.910-922.

Clement, A.C. et al., 1996. An ocean dynamical thermostat. Journal of Climate, 9(9), pp.2190-2196.

Compo, G.P. et al., 2011. The Twentieth Century Reanalysis Project. Quarterly Journal of the Royal Meteorological Society, 137(654), pp.1-28. Available at: http://doi.wiley.com/10.1002/qj.776.

Crowley, T., Zielinski, G. \& Vinther, B., 2008. Volcanism and the little ice age. PAGES News, 16(2), pp.4-5. Available at: http://www.geology.um.maine.edu/publications/Crowley_PAGES_2008.pdf.

Crowley, T.J. \& Unterman, M.B., 2013. Technical details concerning development of a $1200 \mathrm{yr}$ proxy index for global volcanism. Earth System Science Data, 5(1), pp.187-197.

Delworth, T.L., Zhang, R. \& Mann, M.E., 2007. Decadal to Centennial Variability of the Atlantic from Observations and Models. In Ocean Circulation: Mechanisms and Impacts - Past and Future Changes of Meridional Overturning. pp. 131-148.

Ding, Y. et al., 2014. Ocean response to volcanic eruptions in Coupled Model Intercomparison Project 5 simulations. Journal of Geophysical Research: Oceans, 119(9), pp.5622-5637.

Driscoll, S. et al., 2012. Coupled Model Intercomparison Project 5 (CMIP5) simulations of climate following volcanic eruptions. Journal of Geophysical Research: Atmospheres, 117(17).

Emile-Geay, J. et al., 2013. Estimating central equatorial pacific SST variability over the past millennium. Part I: Methodology and validation. Journal of Climate, 26(7), pp.2302-2328.

Emile-Geay, J. et al., 2008. Volcanoes and ENSO over the past millennium. Journal of Climate, 21(13), pp.3134-3148.

Enfield, D.B., Mestas-Nuñez, A.M. \& Trimble, P.J., 2001. The Atlantic multidecadal oscillation and its relation to rainfall and river flows in the continental US. Geophysical Research Letters, 28(10), pp.2077-2080. Available at: http://onlinelibrary.wiley.com/doi/10.1029/2000GL012745/full.

Escudier, R., Mignot, J. \& Swingedouw, D., 2013. A 20-year coupled ocean-sea ice-atmosphere variability mode in the North Atlantic in an AOGCM. Climate Dynamics, 40(3-4), pp.619-636.

Fernández-Donado, L. et al., 2013. Large-scale temperature response to external forcing in simulations and reconstructions of the last millennium. Climate of the Past, 9(1), pp.393-421. Available at: http://www.clim-past.net/9/393/2013/.

Ferry, N. et al., 2010. Mercator global eddy permitting ocean SPPT perturbations in EC-Earth3: impact on seasonal forecast quality. Quarterly Newsletter, 36(Mercator Ocean), p.3439.

Fischer, E.M. et al., 2007. European climate response to tropical volcanic eruptions over the last half millennium. Geophysical Research Letters, 34(5), p.L05707. Available at: http://doi.wiley.com/10.1029/2006GL027992.

Gao, C., Robock, A. \& Ammann, C., 2008. Volcanic forcing of climate over the past 1500 years: An improved ice core-based index for climate models. Journal of Geophysical Research: Atmospheres, 113(23), pp.115 .

Gerber, E.P. et al., 2012. Assessing and understanding the impact of stratospheric dynamics and variability on the earth system. Bulletin of the American Meteorological Society, 93(6), pp.845-859.

Graf, H. et al., 1999. Effect of environmental conditions on volcanic plume rise. J Geophys Res, 104(D20), 
pp.24309-24320.

Graf, H.F. et al., 2014. Observational constraints on the tropospheric and near-surface winter signature of the Northern Hemisphere stratospheric polar vortex. Climate Dynamics, pp.1-22.

Gray, S.T. et al., 2004. A tree-ring based reconstruction of the Atlantic Multidecadal Oscillation since 1567 A.D. Geophysical Research Letters, 31(12), p.L12205. Available at: http://doi.wiley.com/10.1029/2004GL019932.

Groisman, P.. Y., 1985. Regional Climatic Consequences of Volcanic Eruptions (in Russian). Meteorologiyai Gidrologiya, 4, pp.39-45.

Guilyardi, E., 2006. El Niño-mean state - Seasonal cycle interactions in a multi-model ensemble. Climate Dynamics, 26(4), pp.329-348.

Hansen, J. et al., 2010. Global surface temperature change. Rev. Geophys., 48(4), p.RG4004. Available at: http://dx.doi.org/10.1029/2010RG000345.

Hazeleger, W. et al., 2012. EC-Earth V2.2: Description and validation of a new seamless earth system prediction model. Climate Dynamics, 39, pp.2611-2629.

Herzog, M. et al., 1998. The effect of phase changes of water on the development of volcanic plumes. $J$ Volcanol Geoth Res, 87, pp.55-74.

Highwood, E.J. \& Stevenson, D.S., 2003. Atmospheric impact of the 1783\&ndash;1784 Laki Eruption: Part II Climatic effect of sulphate aerosol. Atmospheric Chemistry and Physics Discussions, 3(2), pp.1599-1629.

Hurrell, J.W., 1994. Decadal Trends in the North Atlantic Oscillation : Regional Temperatures and Precipitation., (24).

Iwi, A.M. et al., 2012. Mechanisms linking volcanic aerosols to the Atlantic meridional overturning circulation. Journal of Climate, 25(8), pp.3039-3051.

Jin, F.-F., 1997. An Equatorial Ocean Recharge Paradigm for ENSO. Part II: A Stripped-Down Coupled Model. Journal of the Atmospheric Sciences, 54(7), pp.830-847.

Jones, G.S. et al., 2005. An \{AOGCM\} simulation of the climate response to a volcanic super-eruption. Clim. Dyn., 25, pp.725-738.

Jones, P.D., Jonsson, T. \& Wheeler, D., 1997. Extension to the North Atlantic oscillation using early instrumental pressure observations from Gibraltar and south-west Iceland. International Journal of Climatology, 17(13), pp.1433-1450. Available at: http://doi.wiley.com/10.1002/(SICI)10970088(19971115)17:13<1433::AID-JOC203>3.0.CO;2-P.

Jungclaus, J.H., Lohmann, K. \& Zanchettin, D., 2014. Enhanced 20th century heat transfer to the Arctic simulated in the context of climate variations over the last millennium. Climate of the Past Discussions, 10(4), pp.2895-2924. Available at: http://www.clim-past-discuss.net/10/2895/2014/.

Knight, J.R. et al., 2005. A signature of persistent natural thermohaline circulation cycles in observed climate. , 32 , pp.2-5.

Knudsen, M.F. et al., 2014. Evidence for external forcing of the Atlantic Multidecadal Oscillation since termination of the Little Ice Age. Nature communications, 5, p.3323.

Kodera, K., 1994. Influence of volcanic eruptions on the troposphere through stratospheric dynamical processes in the northern hemisphere winter. J. Geophys. Res., 99(D1), pp.1273-1282. Available at: http://dx.doi.org/10.1029/93JD02731.

Labitzke, K. \& McCormick, M., 1992. Stratospheric temperature increases due to Pinatubo aerosols. Geophysical Research Letters, 19(2), pp.207-210.

Lamb, H.H., 1970. Volcanic Dust in the Atmosphere; with a Chronology and Assessment of Its Meteorological Significance. Philosophical Transactions of the Royal Society of London, Series A, 266(1178), pp.425-533.

Landrum, L. et al., 2013. Last Millennium Climate and Its Variability in CCSM4. Journal of Climate, 26(4), pp.1085-1111. Available at: http://journals.ametsoc.org/doi/abs/10.1175/JCLI-D-11-00326.1.

Larkin, N.K. \& Harrison, D.E., 2005. Global seasonal temperature and precipitation anomalies during E1 Ni??o autumn and winter. Geophysical Research Letters, 32(16), pp.1-4.

Lavigne, F. et al., 2013. Source of the great A.D. 1257 mystery eruption unveiled, Samalas volcano, Rinjani Volcanic Complex, Indonesia. Proceedings of the National Academy of Sciences, 110(42), pp.1674216747. Available at: http://www.pnas.org/content/110/42/16742.abstract.

LeGrande, A.N., Tsigaridis, K. \& Bauer, S.E., 2016. Role of atmospheric chemistry in the climate impacts of stratospheric volcanic injections. Nature Geoscience, 9(9), pp.652-655. Available at: http://dx.doi.org/10.1038/ngeo2771.

Lehner, F. et al., 2016. The importance of ENSO phase during volcanic eruptions for detection and attribution. Geophysical Research Letters, 43(6), pp.2851-2858. Available at: http://doi.wiley.com/10.1002/2016GL067935.

Li, J. et al., 2011. Interdecadal modulation of El Niño amplitude during the past millennium. Nature Climate Change, 1(2), pp.114-118. Available at: http://dx.doi.org/10.1038/nclimate1086.

Lohmann, K. et al., 2015. Using simulations of the last millennium to understand climate variability seen in 
paleo-observations: similar variation of Iceland-Scotland overflow strength and Atlantic Multidecadal Oscillation. Climate of the Past Discussions, 10(4), pp.3255-3302. Available at: http://www.clim-pastdiscuss.net/10/3255/2014/.

Luterbacher, J., 2004. European Seasonal and Annual Temperature Variability, Trends, and Extremes Since 1500. Atmospheric Science Letters, 303(1-4), pp.1499-1503. Available at: http://www.sciencemag.org/cgi/doi/10.1126/science.1093877.

Luterbacher, J. \& Pfister, C., 2015. The year without a summer. Nature Geoscience, 8(4), pp.246-248. Available at: http://www.nature.com/doifinder/10.1038/ngeo2404.

Maher, N. et al., 2015. Effects of volcanism on tropical variability. Geophysical Research Letters, p.n/a-n/a. Available at: http://doi.wiley.com/10.1002/2015GL064751.

Mann, M.E., Fuentes, J.D. \& Rutherford, S., 2012. Underestimation of volcanic cooling in tree-ring-based reconstructions of hemispheric temperatures. Nature Geoscience, 5(3), pp.202-205.

Marshall, G.J., 2003. Trends in the Southern Annular Mode from observations and reanalyses. Journal of Climate, 16(24), pp.4134-4143.

Martin, E.R. \& Thorncroft, C.D., 2014. The impact of the AMO on the West African monsoon annual cycle. Quarterly Journal of the Royal Meteorological Society, 140(678), pp.31-46.

McCarthy, G.D. et al., 2015. Ocean impact on decadal Atlantic climate variability revealed by sea-level observations. Nature, 521(7553), pp.508-510. Available at: http://www.nature.com/doifinder/10.1038/nature14491.

McGregor, S. \& Timmermann, A., 2011. The effect of explosive tropical volcanism on ENSO. Journal of Climate, 24(8), pp.2178-2191.

McGregor, S., Timmermann, A. \& Timm, O., 2010. A unified proxy for ENSO and PDO variability since 1650. Climate of the Past, 6, pp.1-17.

Meehl, G. a. et al., 2014. Decadal climate prediction an update from the trenches. Bulletin of the American Meteorological Society, 95(2), pp.243-267.

Menary, M.B. et al., 2015. Exploring the impact of CMIP5 model biases on the simulation of North Atlantic decadal variability.

Mignot, J. et al., 2011. Volcanic impact on the Atlantic Ocean over the last millennium. Climate of the Past, 7(4), pp.1439-1455.

Miller, G.H. et al., 2012. Abrupt onset of the Little Ice Age triggered by volcanism and sustained by seaice/ocean feedbacks. Geophysical Research Letters, 39(2), pp.1-5.

Morice, C.P. et al., 2012. Quantifying uncertainties in global and regional temperature change using an ensemble of observational estimates: The HadCRUT4 data set. Journal of Geophysical Research Atmospheres, $117(8)$.

Newhall, C.G. \& Self, S., 1982. The volcanic explosivity index (VEI) an estimate of explosive magnitude for historical volcanism. Journal of Geophysical Research, 87(C2), p.1231. Available at: http://doi.wiley.com/10.1029/JC087iC02p01231.

Oberhuber, J. et al., 1998. Volcanic plume simulation on large scales. . J Volcanol Geoth Res, 87, pp.29-53.

Ohba, M. et al., 2013. Impact of Strong Tropical Volcanic Eruptions on ENSO Simulated in a Coupled GCM. Journal of Climate, 26(14), pp.5169-5183.

Ortega, P. et al., 2015. A model-tested North Atlantic Oscillation reconstruction for the past millennium. Nature, 523(7558), pp.71-74. Available at: http://www.nature.com/doifinder/10.1038/nature14518.

Ortega, P. et al., 2011. Variability of the Atlantic meridional overturning circulation in the last millennium and two IPCC scenarios. Climate Dynamics, 38(9-10), pp.1925-1947.

Otterå, O.H. et al., 2010. External forcing as a metronome for Atlantic multidecadal variability. Nature Geoscience, 3(10), pp.688-694.

PAGES 2k-PMIP3 group, 2015. Continental-scale temperature variability in PMIP3 simulations and PAGES 2k regional temperature reconstructions over the past millennium. Climate of the Past, 11(12), pp.1673-1699.

Pausata, F.S.R. et al., 2015. Impacts of high-latitude volcanic eruptions on ENSO and AMOC. Proceedings of the National Academy of Sciences, 112(45), pp.13784-13788. Available at: http://www.pnas.org/content/112/45/13784.abstract.

Persechino, A., Mignot, J. \& Swingedouw, D., 2013. Decadal predictability of the Atlantic meridional overturning circulation and climate in the IPSL-CM5A-LR model. Climate dynamics, 40(9-10), pp.23592380.

Philander, S.G., 1999. A review of tropical ocean-atmosphere interactions. Tellus, Series B: Chemical and Physical Meteorology, 51(1 SPEC. ISS.), pp.71-90.

Phipps, S.J. et al., 2013. Paleoclimate data-model comparison and the role of climate forcings over the past 1500 years. Journal of Climate, 26(18), pp.6915-6936.

Rayner, N.A. et al., 2003. Global analyses of sea surface temperature, sea ice, and night marine air temperature since the late nineteenth century. Journal of Geophysical Research, 108(D14), p.4407. Available at: 
http://dx.doi.org/10.1029/2002JD002670\nhttp://doi.wiley.com/10.1029/2002JD002670\nhttp://linkinghub .elsevier.com/retrieve/pii/S1381116906013641.

Robock, A. \& Mao, J., 1992. Winter warming from large volcanic eruptions. Geophysical Research Letters, 19(24), pp.2405-2408.

Robock, a., 2000. Volcanic eruptions and climate. Reviews of Geophysics, 38(2), pp.191-219.

Ruprich-Robert, Y. \& Cassou, C., 2015. Combined influences of seasonal East Atlantic Pattern and North Atlantic Oscillation to excite Atlantic multidecadal variability in a climate model. Climate Dynamics, 44, pp.229-253.

Santer, B.D. et al., 2015. Observed multivariable signals of late 20th and early 21 st century volcanic activity. Geophysical Research Letters, 42, pp.500-509.

Santer, B.D. et al., 2014. Volcanic contribution to decadal changes in tropospheric temperature. Nature Geoscience, 7(3), pp.185-189.

Sapper, K., 1927. Vulkankunde, Stuttgart: Engelhorn Verlag.

Sato, M. et al., 1993. Stratospheric Aerosol Optical Depths, 1850-1990. Journal of Geophysical Research, 98(D12), pp.22987-22994.

Schmidt, G. a. et al., 2012. Climate forcing reconstructions for use in PMIP simulations of the Last Millennium (v1.1). Geoscientific Model Development, 5(1), pp.185-191.

Schnetzler, C.C. et al., 1997. A proposed volcanic sulfur dioxide index (VSI). Journal of Geophysical Research, 102(B9), pp.20087-20091.

Schurer, A.P. et al., 2013. Separating forced from chaotic climate variability over the past millennium. Journal of Climate, 26(18), pp.6954-6973.

Schurer, A.P., Tett, S.F.B. \& Hegerl, G.C., 2014. Small influence of solar variability on climate over the past millennium. Nature Geoscience, 7(2), pp.1-5. Available at: http://www.nature.com/doifinder/10.1038/ngeo2040.

Seager, R., Zebiak, S.E. \& Cane, M.A., 1988. A Model of the Tropical Pacific Sea Surface Temperature Climatology. Journal of Geophysical Research, 93(C2), pp.1265-1280.

Seferian, R. et al., 2014. Multiyear predictability of tropical marine productivity. Proceedings of the National Academy of Sciences, 111(32), pp.11646-11651. Available at: http://www.pnas.org/cgi/doi/10.1073/pnas.1315855111.

Servonnat, J. et al., 2010. Influence of solar variability, CO\&lt;sub\&gt;2\&lt;/sub\&gt; and orbital forcing between 1000 and $1850 \mathrm{AD}$ in the IPSLCM4 model. Climate of the Past, 6(4), pp.445-460. Available at: http://www.clim-past.net/6/445/2010/.

Shindell, D.T. et al., 2003. Volcanic and solar forcing of climate change during the preindustrial era. Journal of Climate, 16(24), pp.4094-4107.

Sicre, M.-A. et al., 2013. Sea surface temperature and sea ice variability in the subpolar North Atlantic from explosive volcanism of the late thirteenth century. Geophysical Research Letters, 40(20), pp.5526-5530. Available at: http://doi.wiley.com/10.1002/2013GL057282 [Accessed November 17, 2014].

Sigl, M. et al., 2015. Timing and climate forcing of volcanic eruptions for the past 2,500 years. Nature. Available at: http://www.nature.com/doifinder/10.1038/nature14565.

Smith, T.M. et al., 2008. Improvements to NOAA's historical merged land-ocean surface temperature analysis (1880-2006). Journal of Climate, 21(10), pp.2283-2296.

Soden, B.J., 2002. Global Cooling After the Eruption of Mount Pinatubo: A Test of Climate Feedback by Water Vapor. Science, 296(5568), pp.727-730. Available at: http://www.sciencemag.org/cgi/doi/10.1126/science.296.5568.727.

Stenchikov, G. et al., 2006. Arctic Oscillation response to volcanic eruptions in the IPCC AR4 climate models. Journal of Geophysical Research: Atmospheres, 111(7), pp.1-17.

Stenchikov, G. et al., 2009. Volcanic signals in oceans. Journal of Geophysical Research: Atmospheres, 114(16), pp.1-13.

Stoffel, M. et al., 2015. Estimates of volcanic-induced cooling in the Northern Hemisphere over the past 1,500 years. Nature Geoscience, (Published online). Available at: http://www.nature.com/doifinder/10.1038/ngeo2526.

Sueyoshi, T. et al., 2013. Set-up of the PMIP3 paleoclimate experiments conducted using an Earth system model, MIROC-ESM. Geoscientific Model Development, 6(3), pp.819-836. Available at: http://www.geoscimodel-dev.net/6/819/2013/.

Sutton, R.T. \& Hodson, D.L.R., 2005. Atlantic Ocean Forcing of North American and European Summer Climate. Science, 309. no. 5(2005), pp.115-118.

Swingedouw, D. et al., 2015. Bidecadal North Atlantic ocean circulation variability controlled by timing of volcanic eruptions. Nature communications, 6, p.6545.

Swingedouw, D. et al., 2011. Natural forcing of climate during the last millennium: Fingerprint of solar variability. Climate Dynamics, 36(7), pp.1349-1364. 
Timmreck, C. et al., 2009. Limited temperature response to the very large AD 1258 volcanic eruption. Geophysical Research Letters, 36(21), pp.5-9.

Timmreck, C. et al., 2009. Limited temperature response too the very large $\{\mathrm{AD}\} 1258$ volcanic eruption. Geophys. Res. Letters, 36, p.L21708.

Timmreck, C., 2012. Modeling the climatic effects of large explosive volcanic eruptions. Wiley Interdisciplinary Reviews: Climate Change, 3(6), pp.545-564.

Toohey, M. et al., 2014. The impact of volcanic aerosol on the Northern Hemisphere stratospheric polar vortex : mechanisms and sensitivity to forcing structure. , pp.16777-16819.

Trenberth, K.E. \& Shea, D.J., 2006. Atlantic hurricanes and natural variability in 2005. Geophysical Research Letters, 33(12), p.L12704.

Trenberth, K.E. \& Stepaniak, D.P., 2001. Indices of El Nino evolution. Journal of Climate, 14(8), pp.1697-1701.

Tung, K.-K. \& Zhou, J., 2010. The Pacific's Response to Surface Heating in 130 Yr of SST: La Niña-like or El Niño-like? Journal of the Atmospheric Sciences, 67(2009), pp.2649-2657.

Vecchi, G.A. \& Wittenberg, A.T., 2010. El Nino and our future climate: where do we stand? Wiley Interdisciplinary Reviews-Climate Change, 1(2), pp.260-270.

Vernier, J.P. et al., 2011. Major influence of tropical volcanic eruptions on the stratospheric aerosol layer during the last decade. Geophysical Research Letters, 38(January 2005), pp.1-8.

Vernier, J.P. et al., 2009. Tropical Stratospheric aerosol layer from CALIPSO Lidar observations. Journal of Geophysical Research D: Atmospheres, 114(24), p.D00H10. Available at: http://doi.wiley.com/10.1029/2009JD011946.

Wallace, J.M. \& Gutzler, D.S., 1981. Teleconnections in the Geopotential Height Field during the Northern Hemisphere Winter. Monthly Weather Review, 109(4), pp.784-812.

Wilson, R. et al., 2010. Reconstructing ENSO: the influence of method, proxy data, climate forcing and teleconnections. Journal of Quaternary Science, 25(1), pp.62-78.

World Meteorological, 2014. El niño/la niña update, Available at: http://www.wmo.int/pages/prog/wcp/wcasp/enso_updates.html.

Zanchettin, D., Bothe, O., et al., 2013. Background conditions influence the decadal climate response to strong volcanic eruptions. Journal of Geophysical Research: Atmospheres, 118(10), pp.4090-4106.

Zanchettin, D. et al., 2011. Bi-decadal variability excited in the coupled ocean-atmosphere system by strong tropical volcanic eruptions. Clim. Dyn., in press(1-2), pp.419-444.

Zanchettin, D., Timmreck, C., et al., 2013. Delayed winter warming: A robust decadal response to strong tropical volcanic eruptions? Geophysical Research Letters, 40(1), pp.204-209.

Zanchettin, D. et al., 2012. Multidecadal-to-centennial SST variability in the MPI-ESM simulation ensemble for the last millennium. Climate Dynamics, 40(5-6), pp.1301-1318.

Zanchettin, D. et al., 2016. The Model Intercomparison Project on the climatic response to Volcanic forcing (VolMIP): Experimental design and forcing input data. Geoscientific Model Development Discussions, (April), pp.1-33. Available at: http://www.geosci-model-dev-discuss.net/gmd-2016-68/.

Zebiak, S.E. \& Cane, M.A., 1987. A model El Nino-Southern Oscillation. Monthly Weather Review, 115(10), pp.2262-2278. Available at: http://journals.ametsoc.org/doi/pdf/10.1175/15200493\%281987\%29115\%3C2262\%3AAMENO\%3E2.0.CO\%3B2.

Zhang, D., Blender, R. \& Fraedrich, K., 2013. Volcanoes and ENSO in millennium simulations: global impacts and regional reconstructions in East Asia. Theoretical and Applied Climatology, 111(3-4), pp.437-454. Available at: http://dx.doi.org/10.1007/s00704-012-0670-6.

Zhang, R. \& Delworth, T.L., 2006. Impact of Atlantic multidecadal oscillations on India/Sahel rainfall and Atlantic hurricanes. Geophysical Research Letters, 33(17), p.L17712.

Zhang, R. \& Delworth, T.L., 2007. Impact of the Atlantic Multidecadal Oscillation on North Pacific climate variability. Geophysical Research Letters, 34(23), pp.2-7.

Zhong, Y., Miller, G. \& Otto-Bliesner, B., 2011. Centennial-scale climate change from decadally-paced explosive volcanism: a coupled sea ice-ocean mechanism. Climate Dynamics.

Zhou, T. et al., 2011. A comparison of the Medieval Warm Period, Little Ice Age and 20th century warming simulated by the FGOALS climate system model. Chinese Science Bulletin, 56(28-29), pp.3028-3041. 


\section{List of Tables}

\begin{tabular}{|c|c|c|c|c|c|c|}
\hline VEI & $\begin{array}{l}\text { Ejecta } \\
\text { volume }\end{array}$ & Classification & Plume & $\begin{array}{l}\text { Tropospheric } \\
\text { injection }\end{array}$ & $\begin{array}{l}\text { Stratospheric } \\
\text { injection }\end{array}$ & Examples \\
\hline 0 & $\begin{array}{l}<10,000 \\
\mathrm{~m}^{3}\end{array}$ & Hawaiian & $\begin{array}{l}<100 \\
\mathrm{~m}\end{array}$ & negligible & none & $\begin{array}{l}\text { Kīlauea, Piton de la Fournaise, } \\
\text { Erebus }\end{array}$ \\
\hline$\underline{1}$ & $\begin{array}{l}>10,000 \\
\mathrm{~m}^{3}\end{array}$ & $\begin{array}{l}\text { Hawaiian / } \\
\text { Strombolian }\end{array}$ & $\begin{array}{l}100 \mathrm{~m}- \\
1 \mathrm{~km}\end{array}$ & minor & none & $\begin{array}{l}\text { Nyiragongo (2002), Raoul Island } \\
(2006)\end{array}$ \\
\hline$\underline{2}$ & $\begin{array}{l}> \\
1,000,000 \\
\mathrm{~m}^{3}\end{array}$ & $\begin{array}{l}\text { Strombolian / } \\
\text { Vulcanian }\end{array}$ & $1-5 \mathrm{~km}$ & moderate & none & $\begin{array}{l}\text { Unzen (1792), Galeras (1993), } \\
\text { Sinabung (2010) }\end{array}$ \\
\hline$\underline{\mathbf{3}}$ & $\begin{array}{l}> \\
10,000,000 \\
\mathrm{~m}^{3}\end{array}$ & $\begin{array}{l}\text { Vulcanian / } \\
\text { Peléan }\end{array}$ & $\begin{array}{l}3- \\
15 \mathrm{~km}\end{array}$ & substantial & possible & $\begin{array}{l}\text { Nevado del Ruiz (1985), } \\
\text { Soufrière Hills (1995), Nabro } \\
\text { (2011) }\end{array}$ \\
\hline$\underline{4}$ & $>0.1 \mathrm{~km}^{3}$ & $\begin{array}{l}\text { Peléan / } \\
\text { Plinian }\end{array}$ & $\begin{array}{l}10- \\
25 \mathrm{~km}\end{array}$ & substantial & definite & $\begin{array}{l}\text { Mayon (1814), Pelée (1902), } \\
\text { Eyjafjallajökull (2010) }\end{array}$ \\
\hline$\underline{5}$ & $>1 \mathrm{~km}^{3}$ & Plinian & $\begin{array}{l}20- \\
35 \mathrm{~km}\end{array}$ & substantial & significant & $\begin{array}{l}\text { Vesuvius (79), Fuji (1707), } \\
\text { Mount Tarawera (1886), El } \\
\text { Chichón (1982), Puyehue (2011) }\end{array}$ \\
\hline$\underline{6}$ & $>10 \mathrm{~km}^{3}$ & $\begin{array}{l}\text { Plinian / Ultra- } \\
\text { Plinian }\end{array}$ & $>30 \mathrm{~km}$ & substantial & substantial & $\begin{array}{l}\text { Veniaminof (c. } 1750 \text { BC), } \\
\text { Huaynaputina (1600), Laki } \\
\text { (1783), Krakatau (1883), } \\
\text { Pinatubo (1991) }\end{array}$ \\
\hline$\underline{7}$ & $>100 \mathrm{~km}^{3}$ & Ultra-Plinian & $>40 \mathrm{~km}$ & substantial & substantial & $\begin{array}{l}\text { Thera (c. } 1620 \text { BC), Taupo (180), } \\
\text { Samalas (1257), Tambora } \\
(1815)\end{array}$ \\
\hline$\underline{8}$ & $\begin{array}{l}> \\
1,000 \mathrm{~km}^{3}\end{array}$ & Supervolcanic & $>50 \mathrm{~km}$ & vast & vast & $\begin{array}{l}\text { La Garita Caldera }(26.3 \mathrm{Ma}) \text {, } \\
\text { Yellowstone }(640,000 \mathrm{BC}) \text {, Toba } \\
(74,000 \mathrm{BC}) \text {, Taupo }(24,500 \mathrm{BC})\end{array}$ \\
\hline
\end{tabular}

Table 1: Classification of volcanic eruptions depending on their VEI (adapted from Newhall \& Self 1982) as well as some associated characteristics. The shaded yellow lines correspond to the type of volcanic eruptions which are considered in the present study. 


\begin{tabular}{lccccc}
\hline $\begin{array}{l}\text { Name of the } \\
\text { volcanoes }\end{array}$ & Country & $\begin{array}{c}\text { Final } \\
\text { selected } \\
\text { date }\end{array}$ & $\begin{array}{c}\text { Date from Crowley } \\
\text { \& Unterman } \\
(2013)\end{array}$ & $\begin{array}{c}\text { Date from } \\
\text { Gao et al. } \\
(2008)\end{array}$ & $\begin{array}{c}\text { Date from } \\
\text { Sigl et al. } \\
\text { (2015) }\end{array}$ \\
\hline Samalas & Indonesia & $\mathbf{1 2 5 7}^{*}$ & 1258 & 1258 & 1257 \\
Huaynaputina & Peru & $\mathbf{1 6 0 0}^{\dagger}$ & 1600 & 1600 & 1600 \\
Parker & Philippines & $\mathbf{1 6 4 1}^{\dagger}$ & 1641 & 1641 & 1641 \\
Serua & Indonesia & $\mathbf{1 6 9 3}^{\dagger}$ & 1696 & 1693 & 1694 \\
Unknown & & $\mathbf{1 8 0 9}^{+}$ & 1809 & 1809 & 1809 \\
Tambora & Indonesia & $\mathbf{1 8 1 5}^{\dagger}$ & 1816 & 1815 & 1815 \\
Cosiguina & Nicaragua & $\mathbf{1 8 3 5}^{\dagger}$ & 1835 & 1835 & 1834 \\
Krakatau & Indonesia & $\mathbf{1 8 8 3}^{\dagger}$ & 1884 & 1883 & 1884 \\
\hline
\end{tabular}

Table 2: List of eight large volcanic eruptions common to three alternative reconstructions of the volcanic forcing over last millennium. Eruptions occurring in December are counted in the following year, as they took place in its corresponding winter. Only eruptions with a precise dating, either from historical observations ( ${ }^{*}$; Siebert et al. 2010), or constrained using local deposits and historical records (†; Lavigne et al. 2013) have been considered. 


\begin{tabular}{llll}
\hline Model & Time Span & \multicolumn{1}{c}{ Volcanic forcing } & Reference \\
\hline BCC-CSM1-1 & $850-2005$ & Gao et al. (2008) & x \\
CCSM4 & $850-2005$ & Gao et al. (2008) & Landrum et al. (2013) \\
CSIRO-MK3L-1-2 & $851-2005$ & Gao et al. (2008) & Phipps et al. (2013) \\
FGOALS-gl & $1000-1999$ & Crowley et al. (2008) & Zhou et al. (2011) \\
GISS-E2-R & $850-2005$ & Crowley et al. (2008) & Schmidt et al. (2012) \\
IPSL-CM5A-LR & $850-1999$ & Ammann et al. (2007) & Swingedouw et al. (2015) \\
MIROC-ESM & $850-2005$ & Crowley et al. (2008) & Sueyoshi et al. (2013) \\
MPI-ESM-P & $850-2005$ & Crowley et al. (2008) & Jungclaus et al. (2014) \\
CNRM-CM3 & $1001-1999$ & Ammann et al. (2007) & Swingedouw et al. (2011) \\
\hline
\end{tabular}

Table 3: Last millennium simulations considered from the PMIP3 dataset. All these simulations use a volcanic forcing as optical depth depending of latitude and time with resolution lower or equal than monthly. 


\begin{tabular}{|c|c|c|}
\hline Name of the volcanoes & $\begin{array}{l}\text { Months considered in the } \\
\text { composite analysis }\end{array}$ & $\begin{array}{l}\text { Max. of the optical } \\
\text { depth anomaly (from } \\
\text { Ammann et al. 2007) }\end{array}$ \\
\hline Unknown & Jan. 1168 - Nov. 1168 & 0.18 \\
\hline Unknown & Nov. 1176 - Oct. 1178 & 0.44 \\
\hline Unknown & Feb. 1190 & 0.13 \\
\hline Unknown & Sep. 1231 - May 1233 & 0.31 \\
\hline Samalas & Nov. 1257 - Jan. 1260 & 0.87 \\
\hline Unknown & Apr. 1269 - Feb. 1270 & 0.17 \\
\hline Unknown & Mar. 1278 - Nov. 1279 & 0.33 \\
\hline Unknown & Jul. 1286 - Jun.1287 & 0.18 \\
\hline Kuwae (Tonga) & Mar. 1452 - Dec. 1453 & 0.35 \\
\hline Unknown & Nov. 1459 & 0.13 \\
\hline Huaynaputina (Peru) & Jul. 1600 - Aug. 1601 & 0.22 \\
\hline Parker (Philippines) & Apr. 1641 - May 1642 & 0.19 \\
\hline Unknown & Mar. 1809 - Nov. 1810 & 0.33 \\
\hline Tambora (Indonesia) & Mar. 1815 - Apr. 1817 & 0.44 \\
\hline Unknown & Nov. 1830 & 0.13 \\
\hline Cosiguina (Nicaragua) & May 1835 & 0.13 \\
\hline Krakatau (Indonesia) & Nov. 1883 - Aug. 1884 & 0.18 \\
\hline Santa María (Guatemala) & Feb. 1903 & 0.13 \\
\hline Pinatubo (Philippines) & Oct. 1991 & 0.13 \\
\hline
\end{tabular}

Annex Table 1: Volcanic eruptions considered for the composite analysis performed within the CNRM-CM3 last millennium simulation (Swingedouw et al. 2011) in figures 5, 6 and 8. The first column indicates the name of the volcanoes implied in the eruptions, the second the months where the optical depth was larger or equal than 0.13 , corresponding to the maximum of the optical depth anomaly averaged in latitude from Pinatubo eruption according to Ammann et al. (2007) reconstruction. The last column specifies the maximum of the optical depth anomaly recorded in the same reconstruction for each considered eruption. 


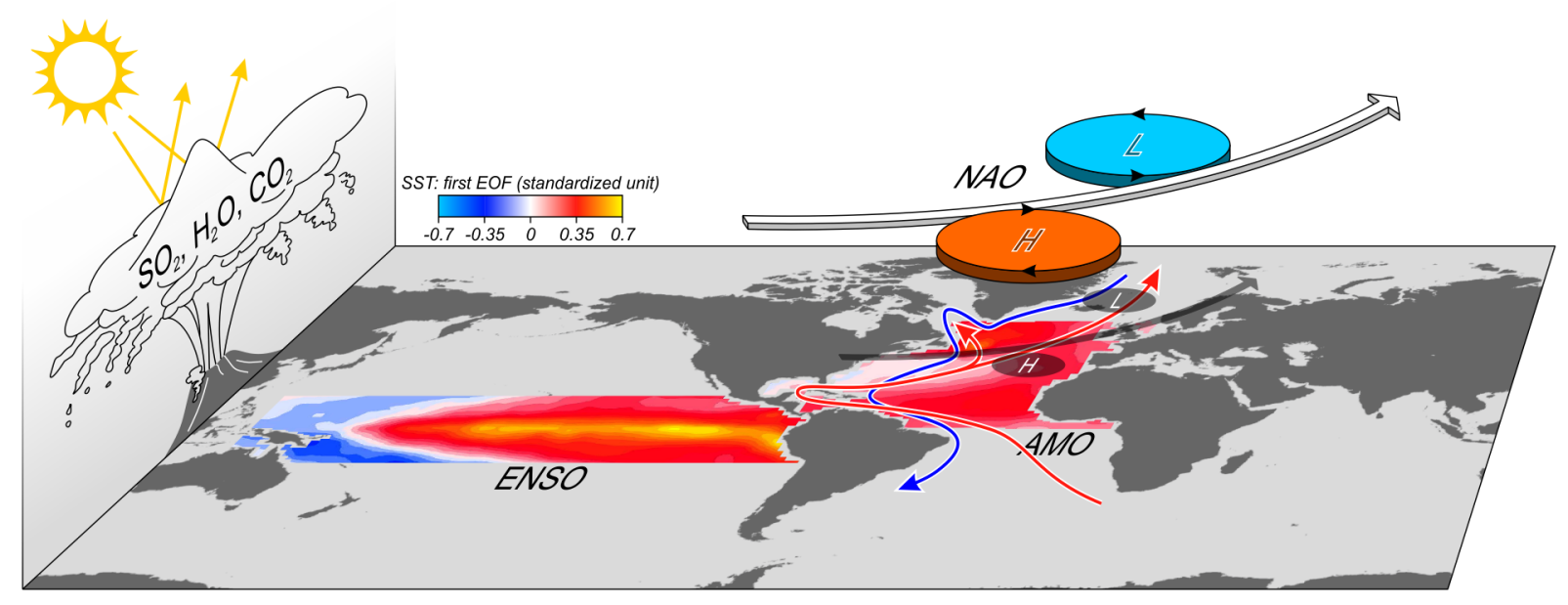

Figure 1: Schematic of the main consequences of volcanic eruptions in the atmosphere and their possible interactions with the main climatic modes. The different features are not at scale and just shown to illustrate the key players discussed in the present paper. The colours represent the first empirical orthogonal function computed from a principal component analysis using HadISST data (Rayner et al. 2003) over the period 1900-2010 for the Tropical Pacific (between $30^{\circ} \mathrm{S}$ and $30^{\circ} \mathrm{N}$ ) and the North Atlantic $\left(0-60^{\circ} \mathrm{N}\right)$ separately, to illustrate the ENSO and AMO modes of variability respectively. The SST data were linearly detrended before performing the principal component analysis. The colour scale represents standardized values over the period 1900-2010. The red and blue arrows in the North Atlantic propose a schematic for the upper warm branch and lower cold branch of the AMOC. The NAO is only represented through a sketch of the anomalous high and low over the North Atlantic, and the anomalous enhanced westerlies associated with it. 


\section{$550 \mathrm{~nm}$ extinction during Pinatubo eruption \\ a) Altitude-time}

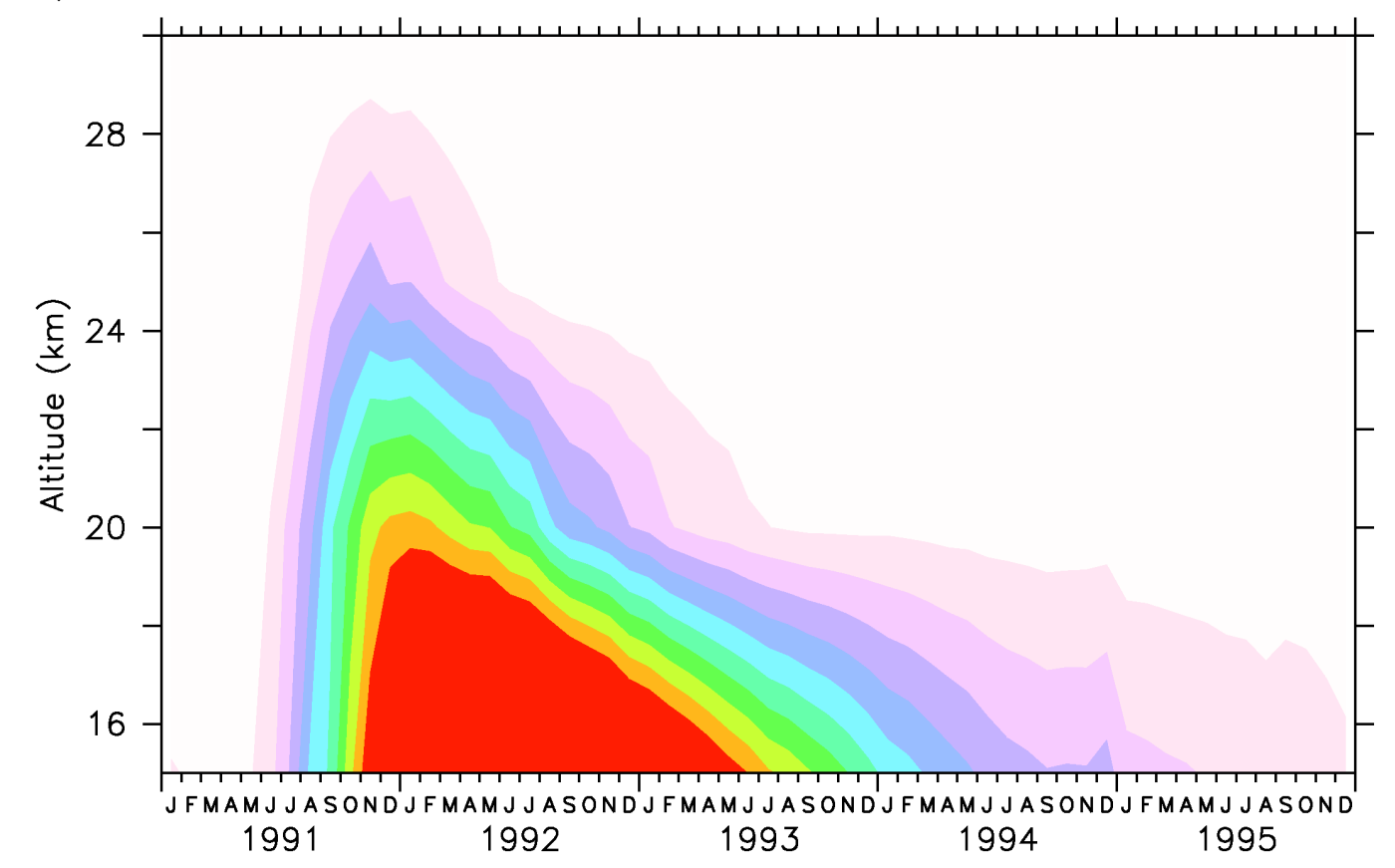

b) Latitude-time

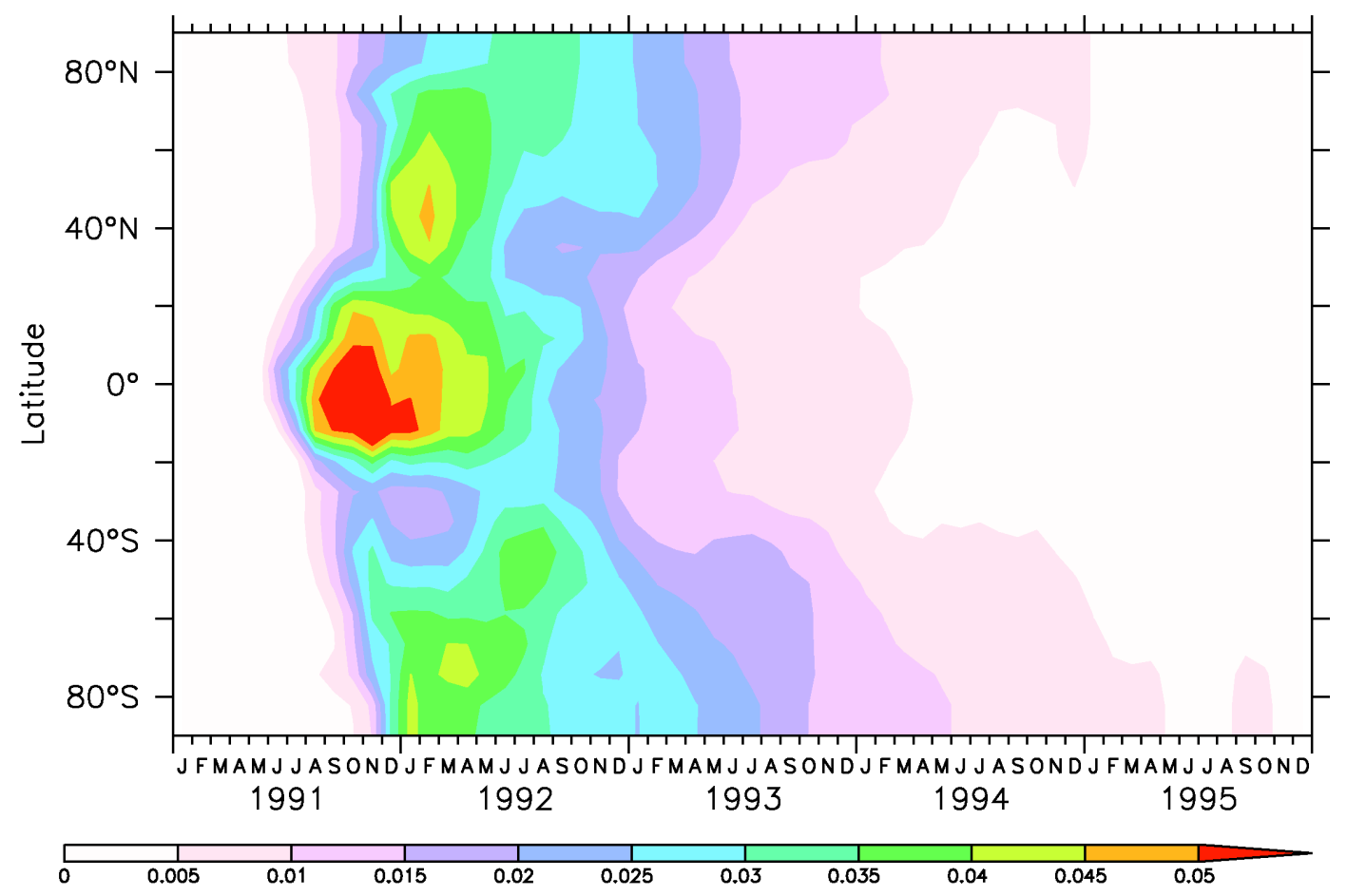

Figure 2 : Variations the aerosol optical extinction at $550 \mathrm{~nm}$ wavelength around the time of the Pinatubo eruption from Sato et al. (1993), a) in altitude-time and b) in latitude-time. 


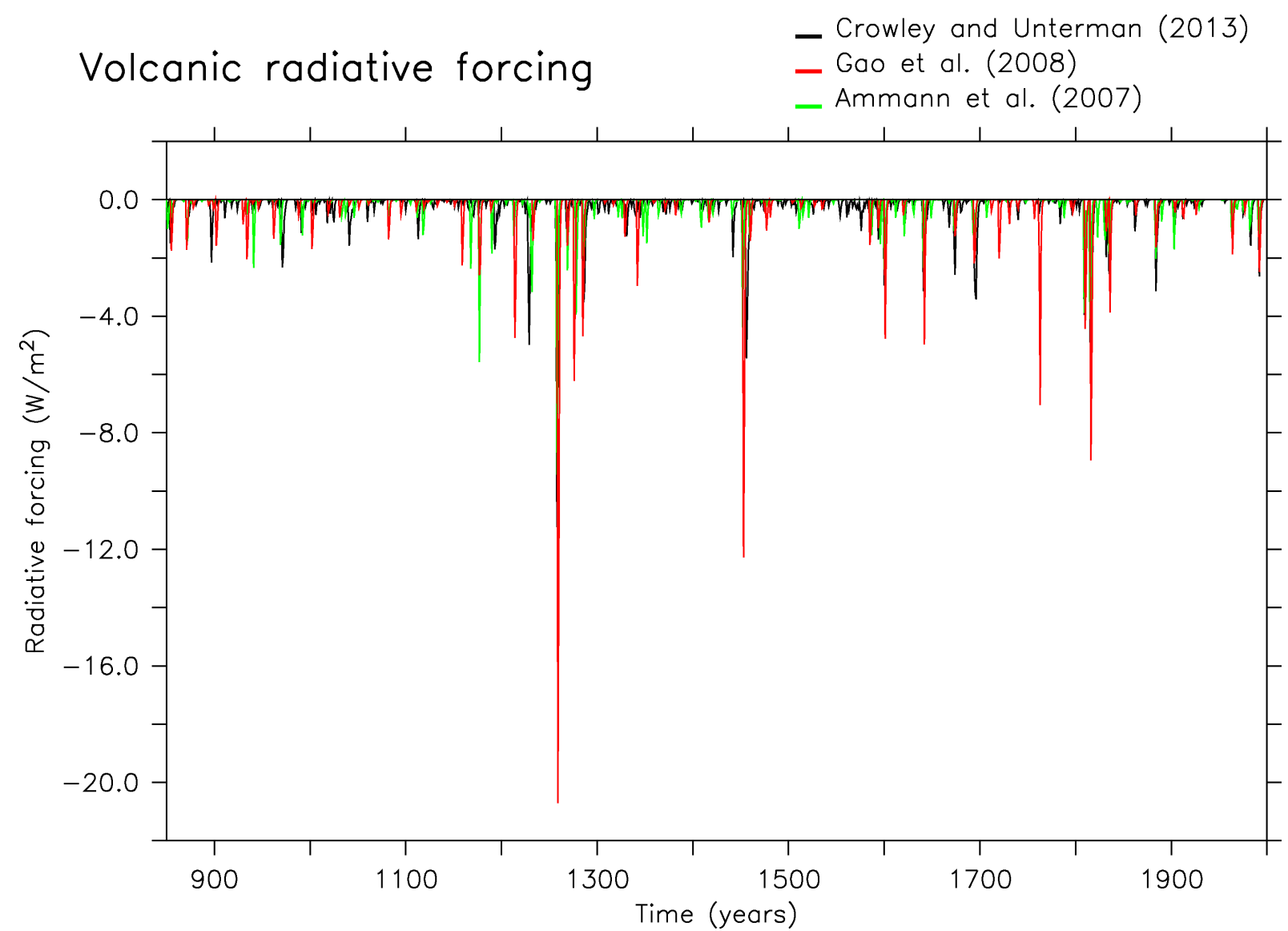

Figure 3 : Reconstruction of change in radiative forcing $\left(\mathrm{W} / \mathrm{m}^{2}\right)$ over the last millennium due to volcanic eruptions for three different estimates: Crowley \& Unterman (2013) in black, Gao et al. (2008) in red and Ammann et al. (2007) in green. 


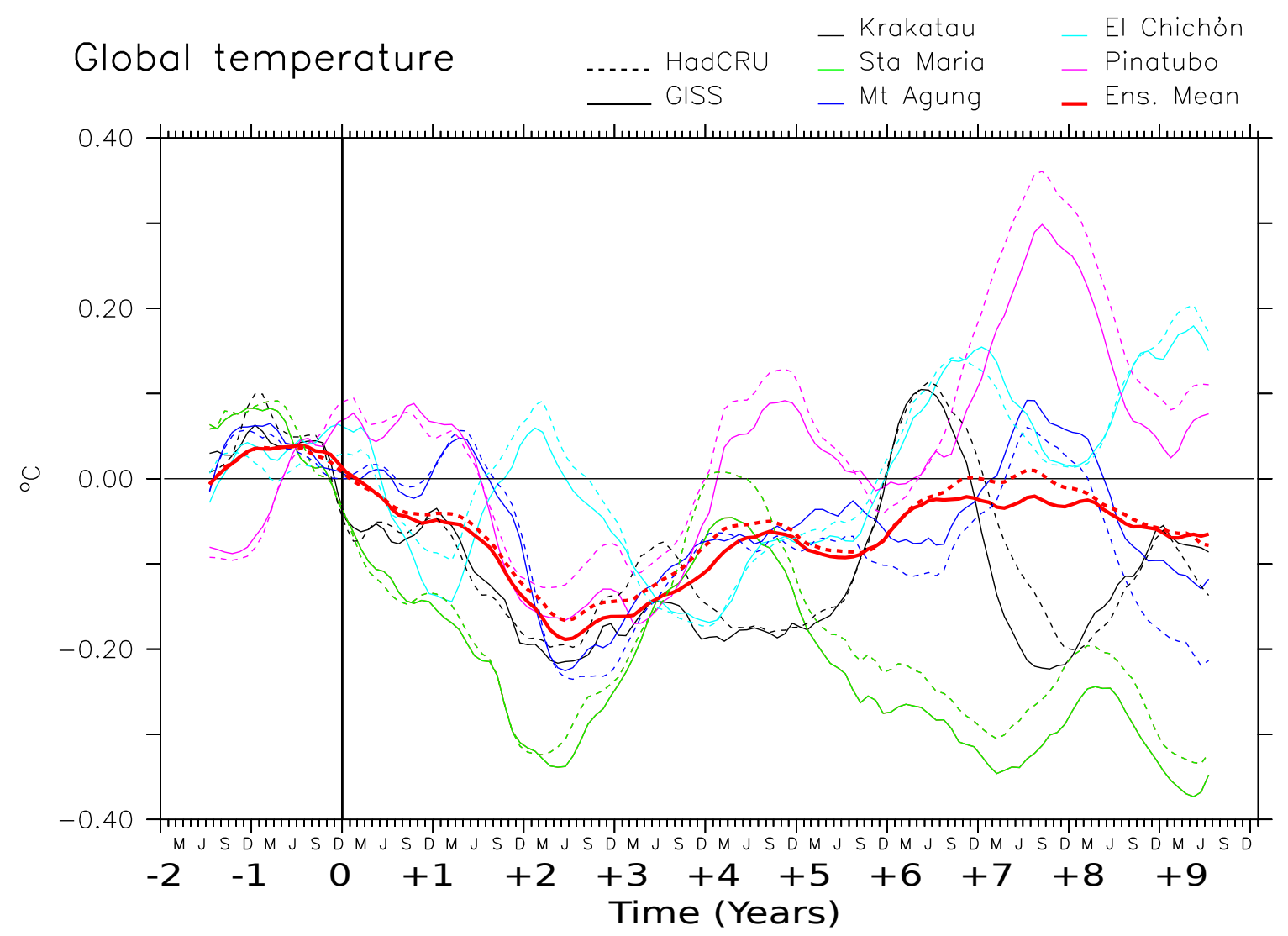

Figure 4: Anomalous global atmospheric temperature at the Earth's surface (in ${ }^{\circ} \mathrm{C}$ ) in response to five major volcanic eruptions over the instrumental era (Krakatau in 1883, Santa María in 1902, Agung in 1963, El Chichón in 1982 and Pinatubo in 1991) from GISS (continuous lines, Hansen et al. 2010) and HadCRU (dashed lines, Morice et al. 2012) datasets. The anomalies refer to the average of the three-year preceding each eruption. The vertical line stands for the year when the onset of the eruptions occurs. The unit of the $\mathrm{x}$-axis is year. 
a) $T 2 m$ composite

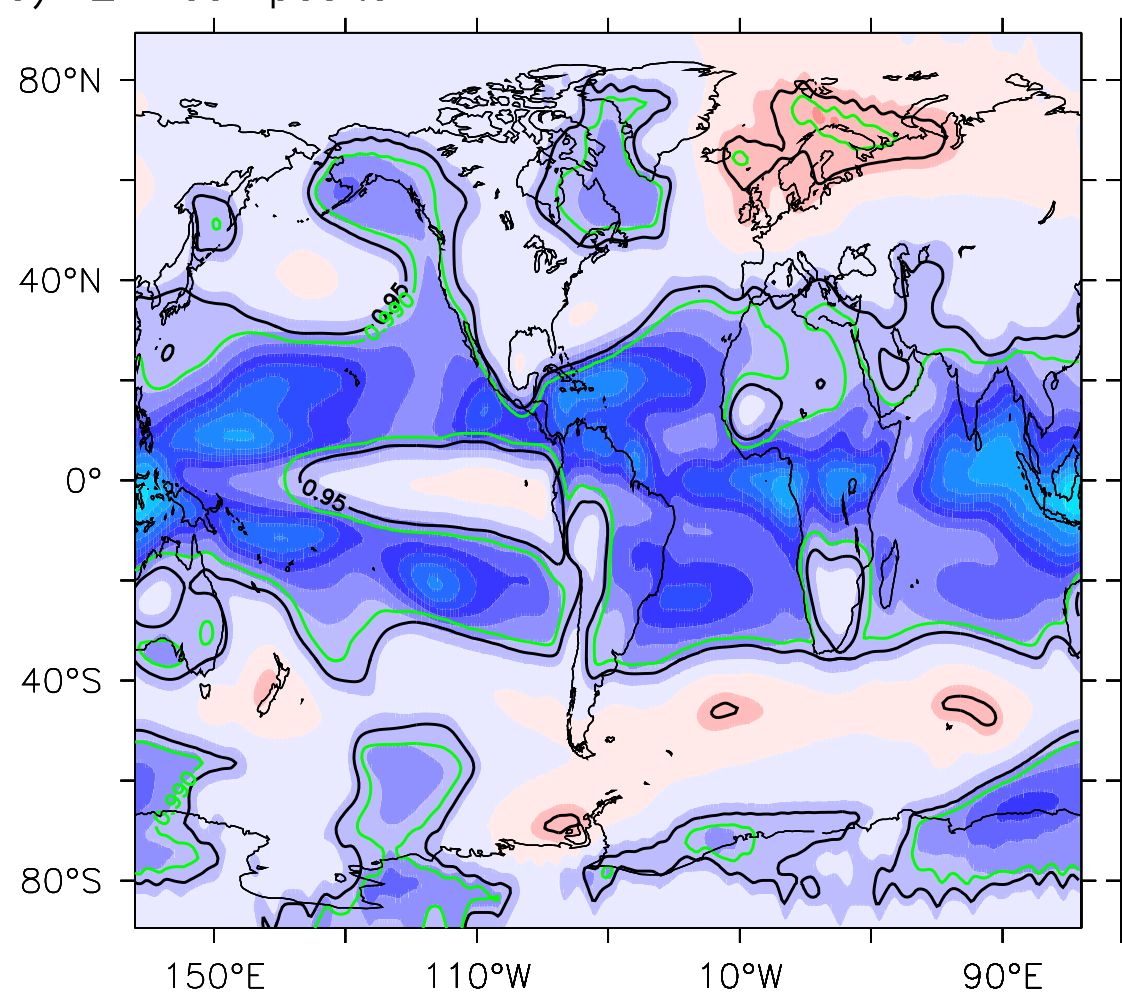

b) Zonal mean

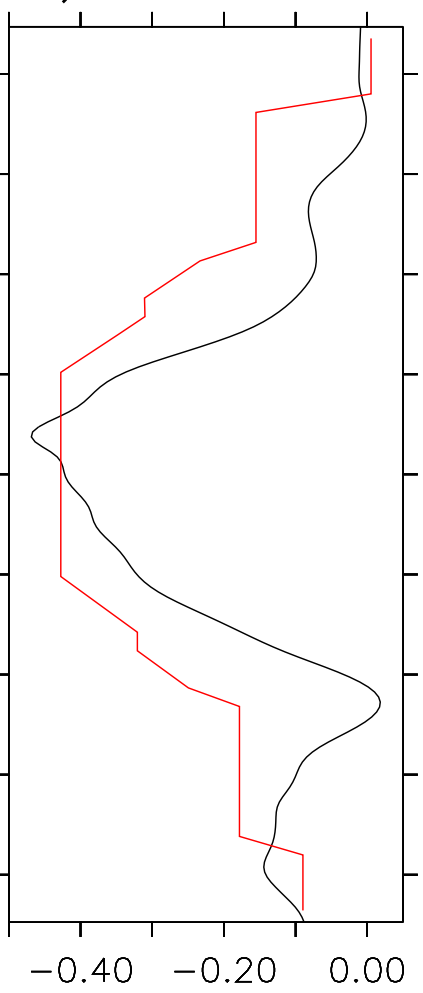

Figure 5 : a) Composite of 2-meter temperature change computed for the 19 eruptions larger or equal than Pinatubo in CNRM-CM3 last millennium simulation in terms of the duration of aerosol imprints in the atmosphere (cf. Annex Table 1). The composite is showing a standardized anomaly with respect to the mean and standard deviation computed at each grid point over the whole simulation and computed for the time frame indicated in Annex Table 1 (i.e. when there is a large enough volcanic aerosol forcing imprints). The black lines indicate the changes within the $90 \%$ level of confidence according to a bootstrap test applied over the whole simulation and the green lines for changes within the 95\% level of confidence. b) Zonal average of composite following the same procedure as in a) for the forcing in terms of optical depth (red line) and composite of the 2-meter temperature (black line). 


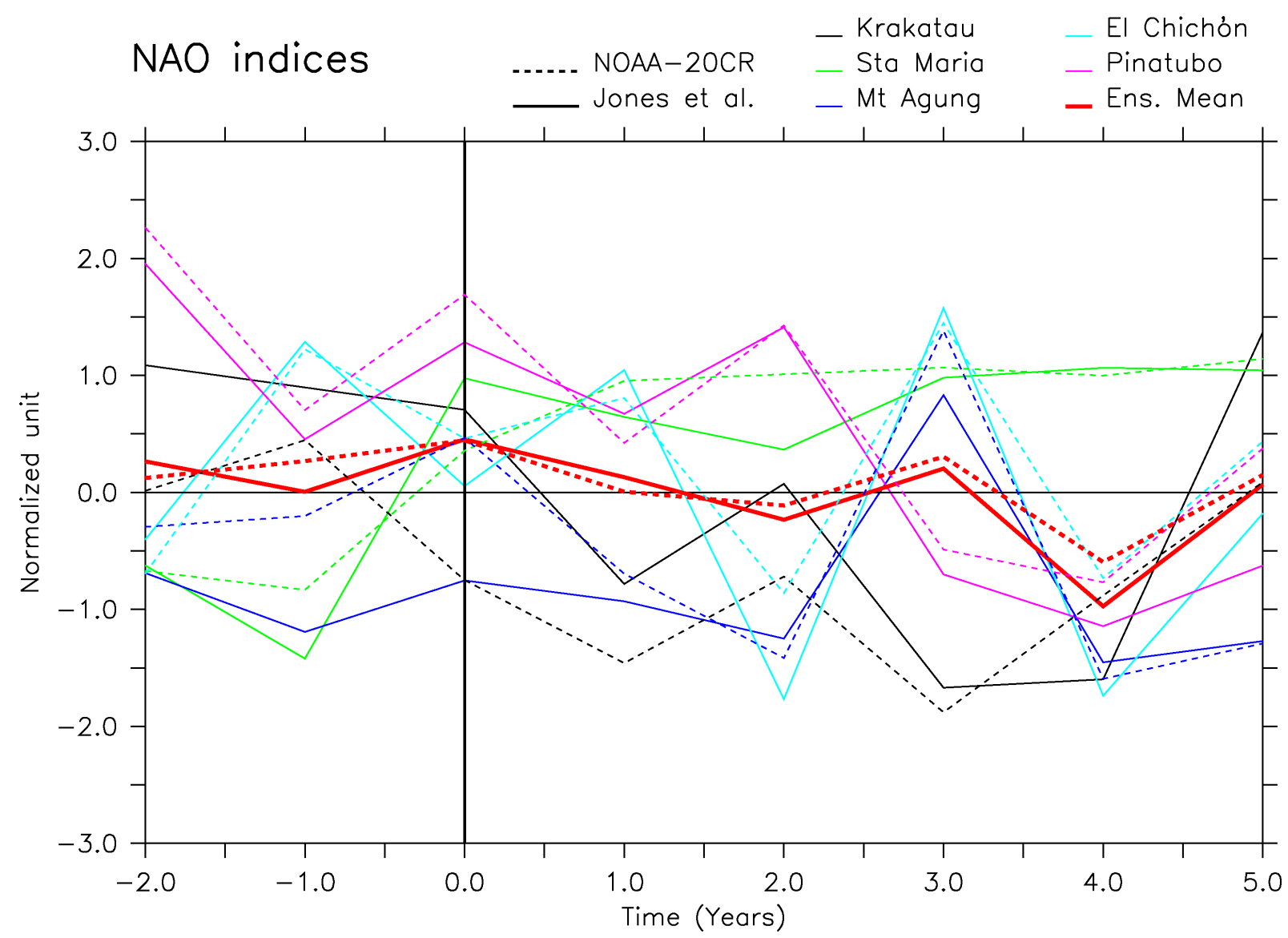

Figure 6: Wintertime (DJF) NAO index for the five largest volcanic eruptions over the instrumental era (Krakatau, Santa María, Agung, El Chichón, Pinatubo) using two different NAO normalized indices: the Gibraltar-Iceland standardised SLP dipole from Jones et al. (1997) in continuous lines and the first empirical orthogonal function of SLP over the North Atlantic $\left(80^{\circ} \mathrm{E}-20^{\circ} \mathrm{W}, 20-80^{\circ} \mathrm{N}\right)$ from the NOAA $20 \mathrm{CR}$ reanalysis (Compo et al. 2011) in dashed lines. The year zero is defined as the first winter beginning after the onset of the eruption (i.e 1883-1884, 1902-1903 1963-1964, 1982-1983, 1991-1992). 

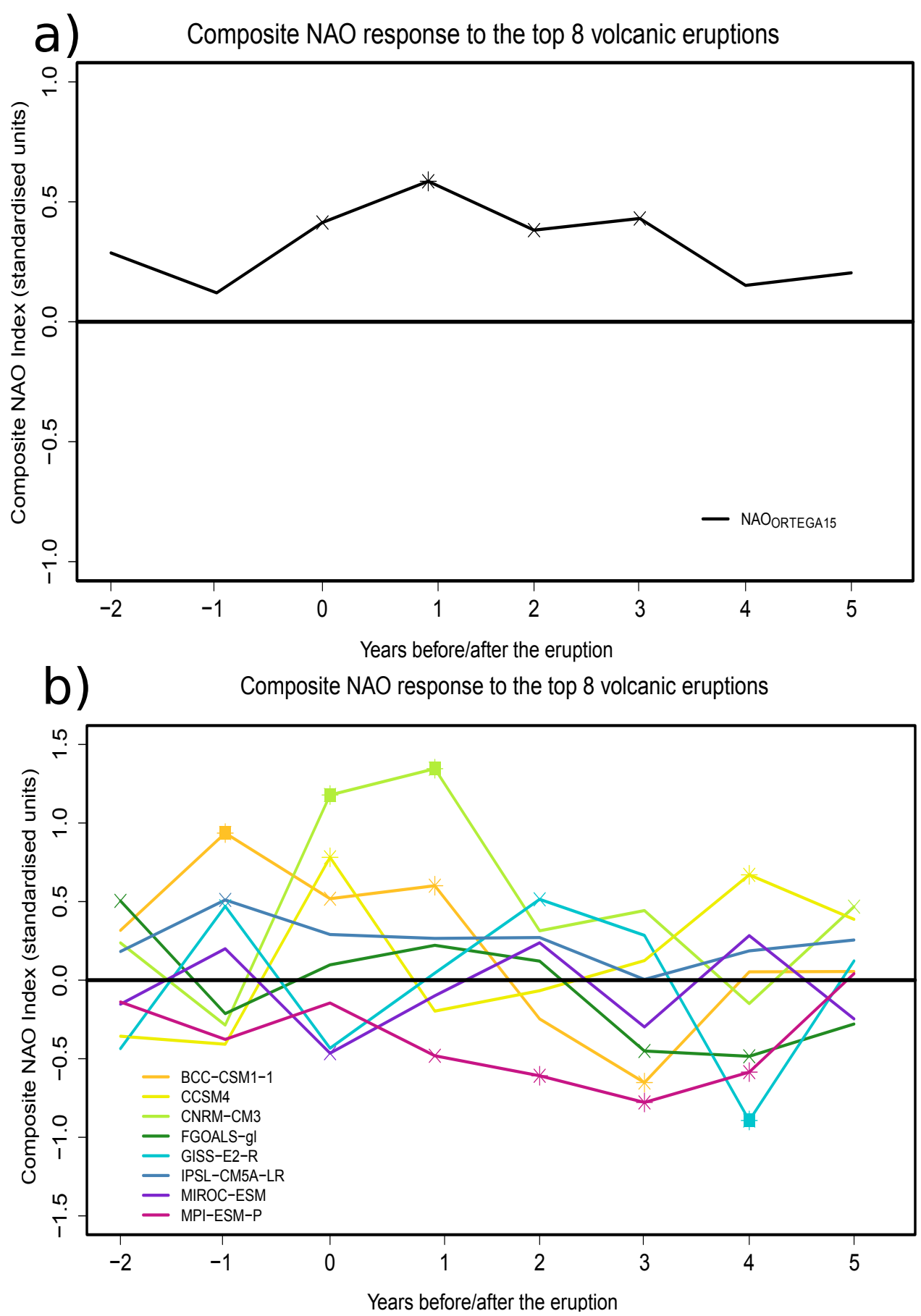

Figure 7: Composite response of winter NAO (in standardised units) to 8 large volcanic eruptions over the period 1000-1900 (same convention for year 0 as in Figure 6) in a) the multiproxy NAO reconstruction from Ortega et al. (2015) and b) a set of 8 PMIP3 last millennium simulations. For the observations, the volcanic eruptions considered took place in 1257, 1600, $1641,1693,1809,1815,1835$ and 1883 ( $c f$. Table 2). For the NAO reconstruction in a), we choose the year 0 one year later than in Ortega et al. (2015) because we consider that the eruptions have low chance to occur in January-February, which is the winter considered by the reconstruction. For the PMIP3 simulations, the 8 largest volcanic eruptions vary depending on the simulation, and the estimates of volcanic forcing used to produce them. All time series are standardised with respect to the full reconstructed or simulated period. Significance is assessed following a Monte-Carlo test based on 1,000 random selections of 8 years from the corresponding NAO time-series. Significant values at the $90 \%, 95 \%$ and $99 \%$ confidence levels are represented by crosses (x), stars $\left(^{*}\right)$ and filled squares $(\mathbf{m})$, respectively. 


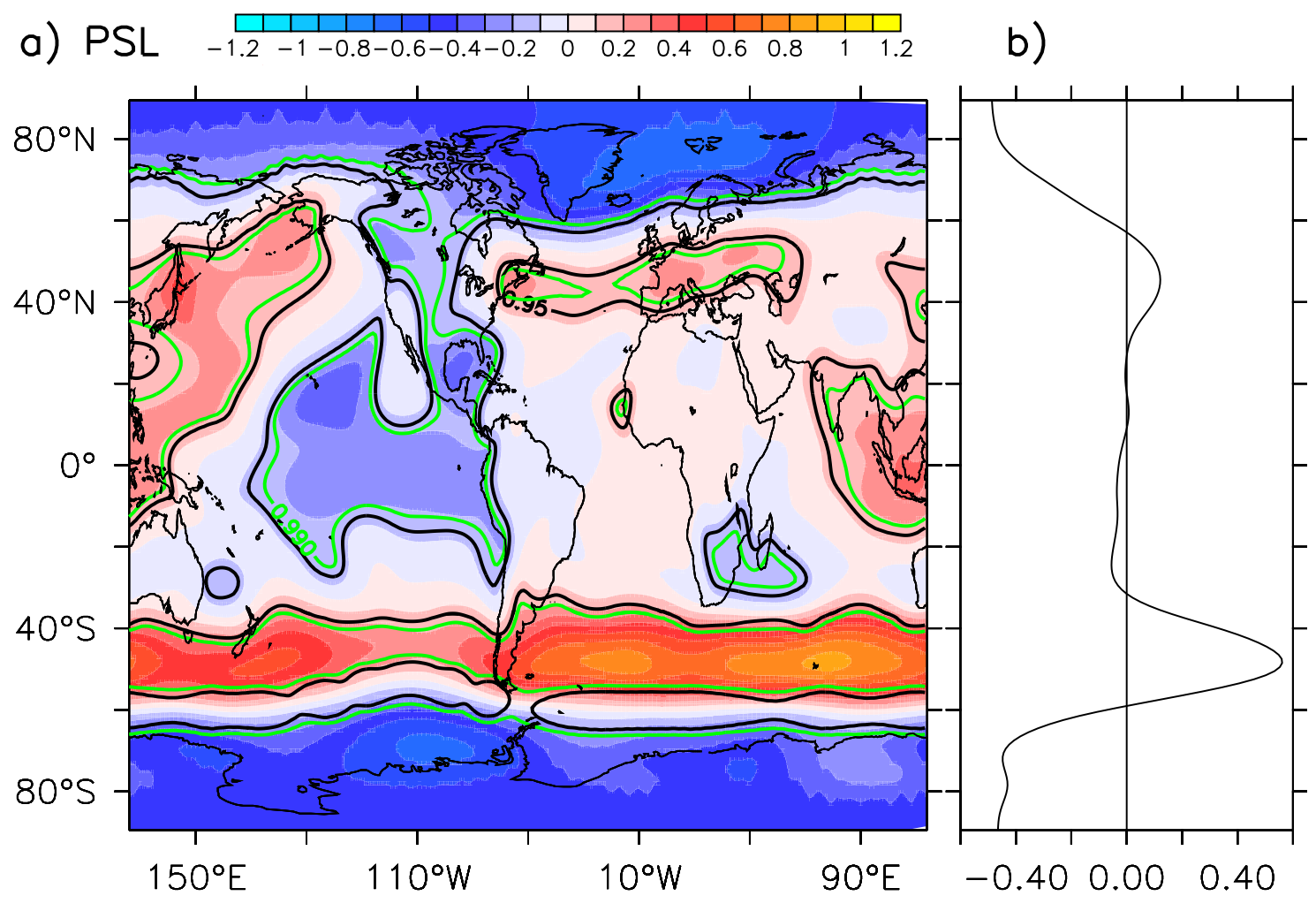

Figure 8: Same as Figure 5 but for a) the composite of sea-level pressure (standardized anomalies with respect to the whole CNRM-CM3 simulation, b) zonal average of the composite. 


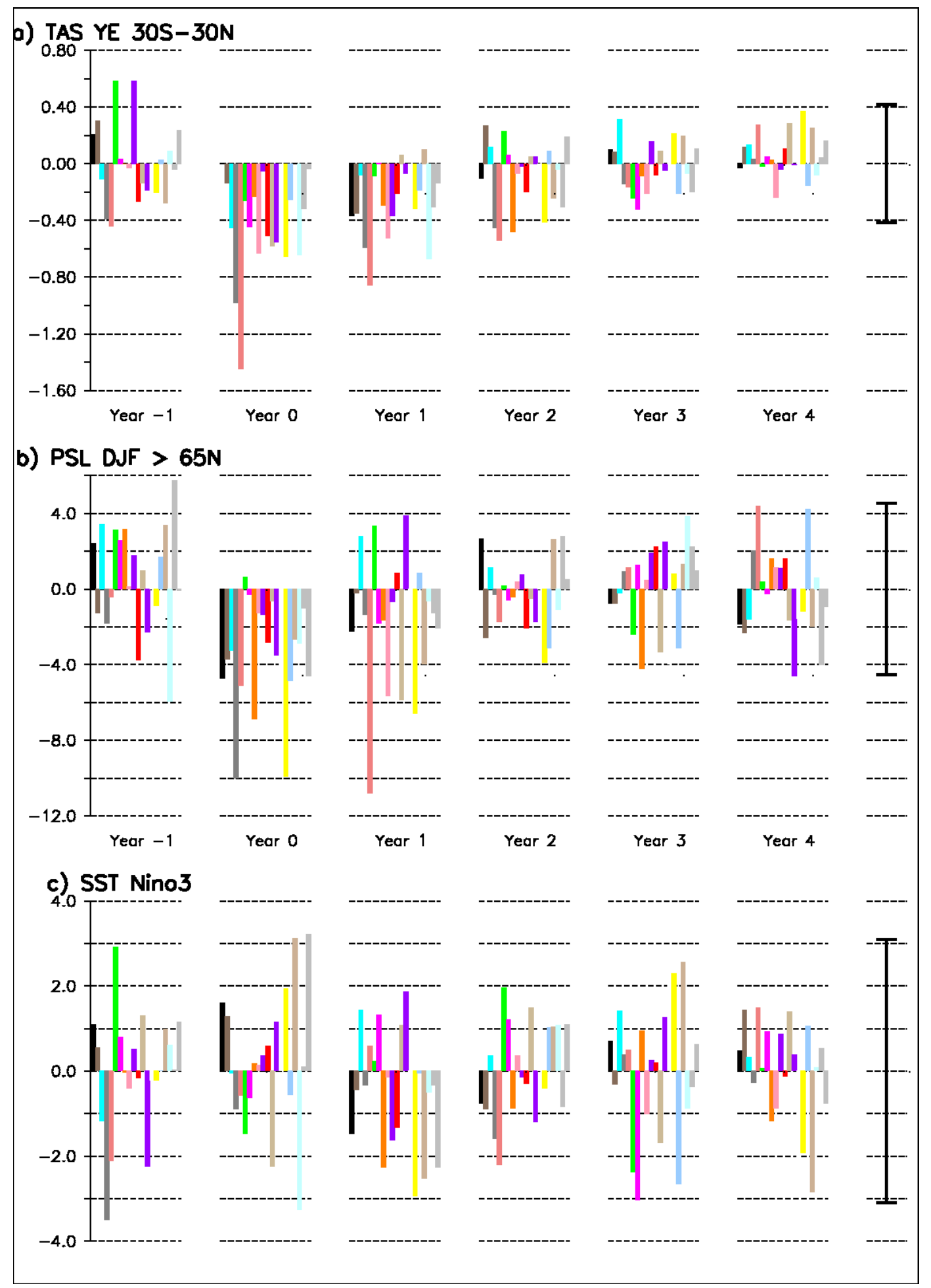

Figure 9: Response to the 19 largest eruptions over the period 1001-2000 (cf. Annex Table 1) in the CNRM-CM3 last millennium simulation for a) the annual mean tropical temperature between $30^{\circ} \mathrm{S}$ and $30^{\circ} \mathrm{N}$ in ${ }^{\circ} \mathrm{C}$, b) the mean sea-level pressure north of $65^{\circ} \mathrm{N}$ in $\mathrm{hPa}$ (a good proxy of Arctic Oscillation and NAO as well), and c) the mean sea-surface temperature in ${ }^{\circ} \mathrm{C}$ in the Niño3 box (a good proxy of ENSO activity). From left to right, panels represent the year before the eruption, the year of the eruption (year 0) and the first four years following the eruption. Each color bar corresponds to the annual mean following one of the 19 eruptions. The whiskers in the right panels stand for \pm 2 std computed from a control simulation with the same model. 
(a)

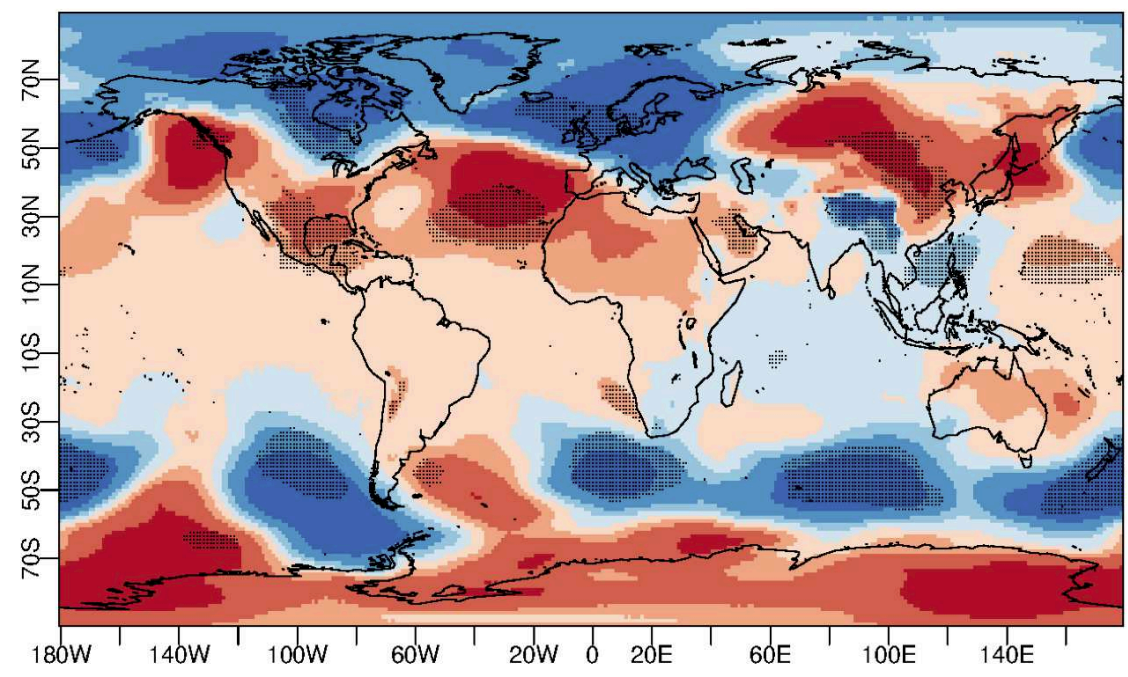

(b)

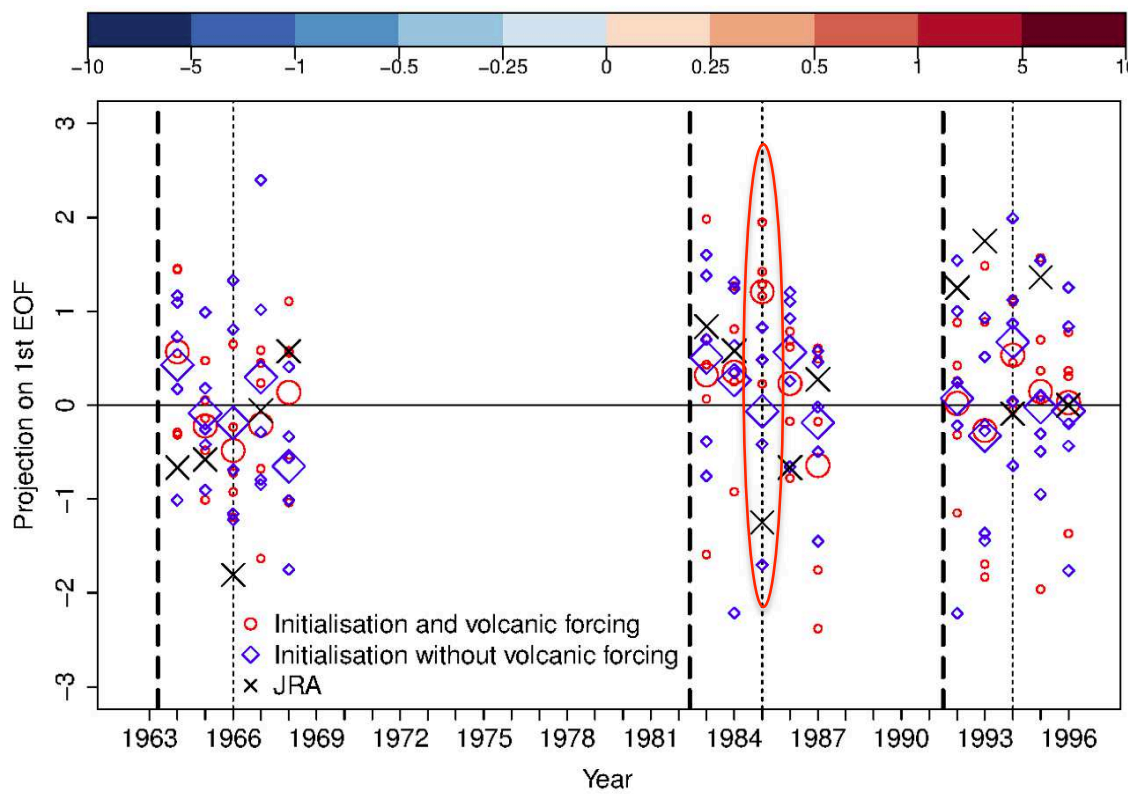

Figure 10: Volcanic impact on EC-Earth hindcast system for the prediction of SLP and NAO variations: (a) SLP difference in hPa between forecasts including and excluding the volcanic forcing, for the third winter on average after the three last eruptions (Agung, 1963; El Chichón, 1982; Pinatubo, 1991), in simulations initialised on November, the year of the eruption (see text for more details on the design of the experiments); hatching stands for significance assessed from the difference between 5-member forecasts at the 95\% level (using a bootstrap resampling). (b) Winter NAO forecast three years in advance. The NAO index is defined as a projection of the SLP on its first EOF, normalized by its standard deviation. Red circles show hindcasts including the GISS volcanic forcing based on observations, whereas blue diamonds show NAO forecasts without any volcanic forcing. Large symbols show the average of the 5 members performed in each hindcast simulation. The red ellipse shows NAO index predicted the third winter following the 1982 El Chichón eruption, positive when including the volcanic forcing (red symbol), neutral without the volcanic forcing (blue symbol) and negative in the Japanese Reanalysis (JRA, Kobayashi et al., 2015) (black cross). There is no discernible difference of NAO index between the forecasts including and excluding the volcanic forcing for winters following the 1963 Agung and the 1991 Pinatubo eruption. 


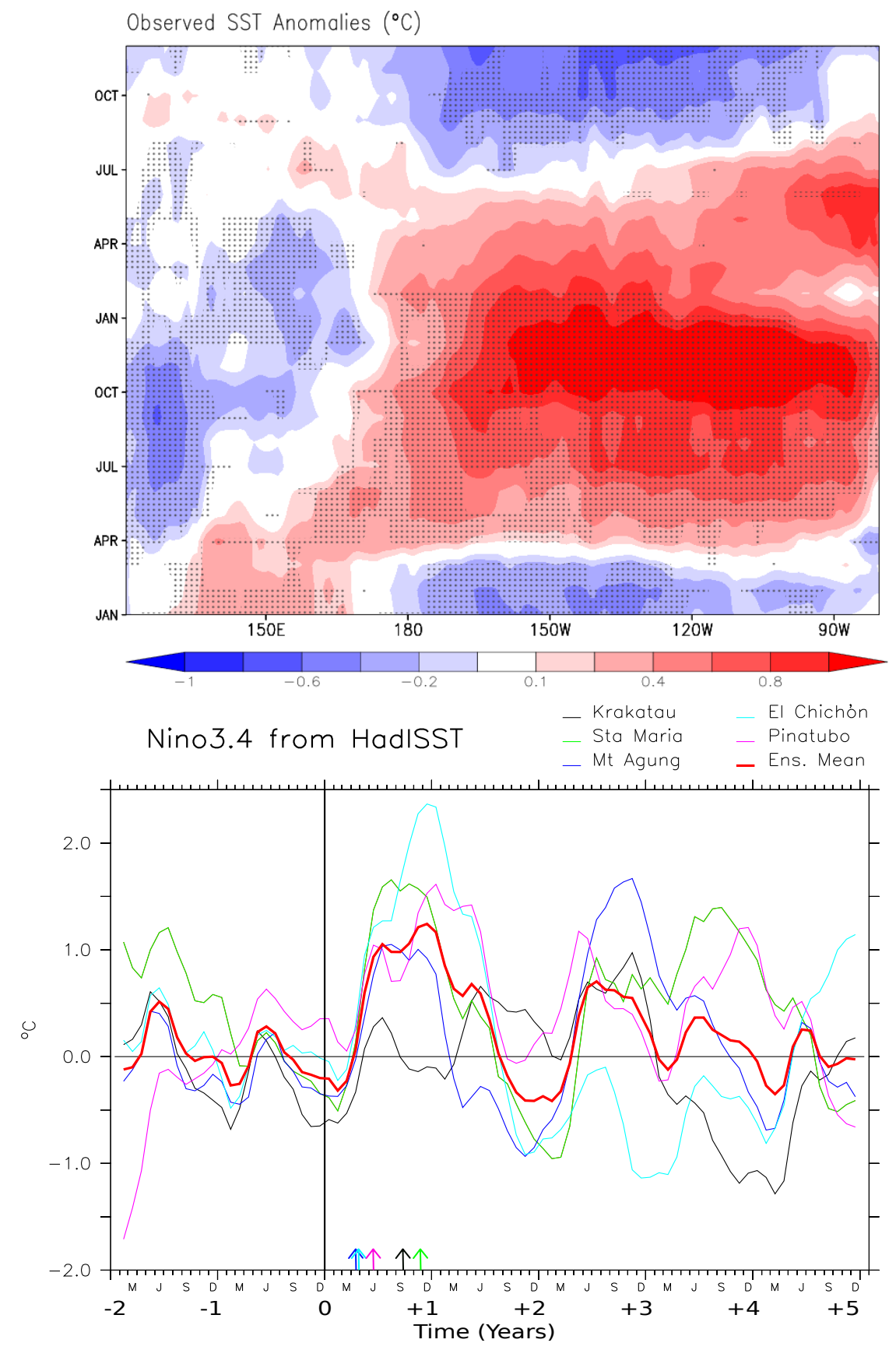

Figure 11: a) Tropical $\left(5^{\circ} \mathrm{S}-5^{\circ} \mathrm{N}\right)$ time-longitude section of composite of the five largest tropical explosive volcanic events of the instrumental era for equatorial Pacific SST anomalies $\left({ }^{\circ} \mathrm{C}\right)$ in HadISST observations (Rayner et al. 2003). SST anomalies are computed with respect to the mean climatology of the 5 years preceding each eruption and smoothed with a three-months hanning filter. The stippling indicates regions and periods for which at least 4 out of 5 of the individual events have consistent SST anomalies. b) Observed SST anomalies from HadISST over Niño3.4 region for the five individual eruptions in a). The ensemble mean is also shown in red. The month 0 corresponds to the first month of January the year of the eruption onset. The mean seasonal cycle computed over the period 1870-2010 have been removed from the time series. A 3 -month running mean has been applied to all data. The colored arrows indicate the month that each eruption occurs. 

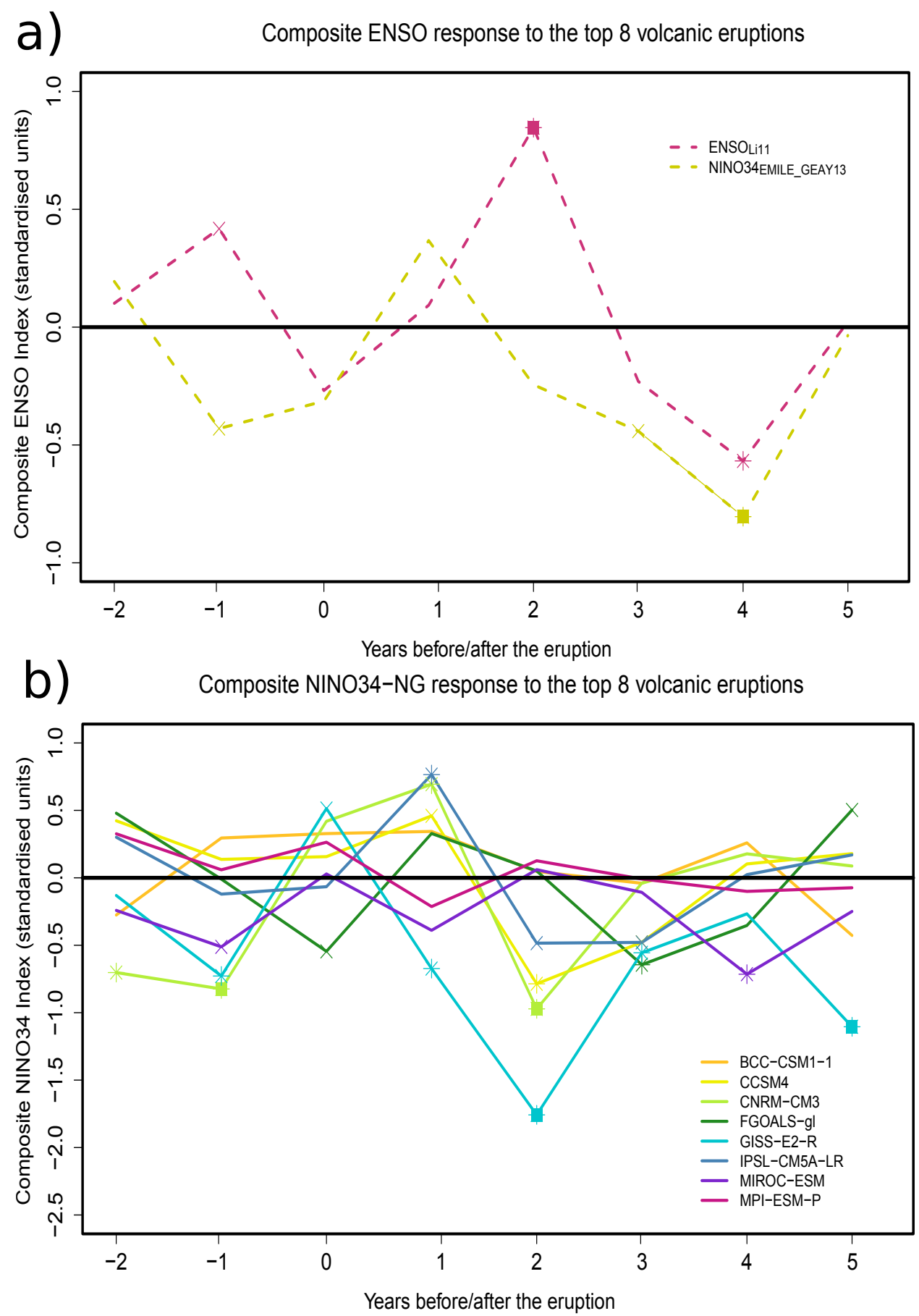

Figure 12 : a) The same as in Figure 7a but for two ENSO reconstructions by Zhou et al. (2011) in pink and Emile-Geay et al. (2013) in green; b) The same as in Figure 7b but for an ENSO index defined as the SST average over the Niño3.4 region. To only look at ENSO-related changes in temperature, the mean global temperature anomaly (which may represent the radiatively forced signal) has been removed from the local average. All ENSO time series are standardised as in7 9. 

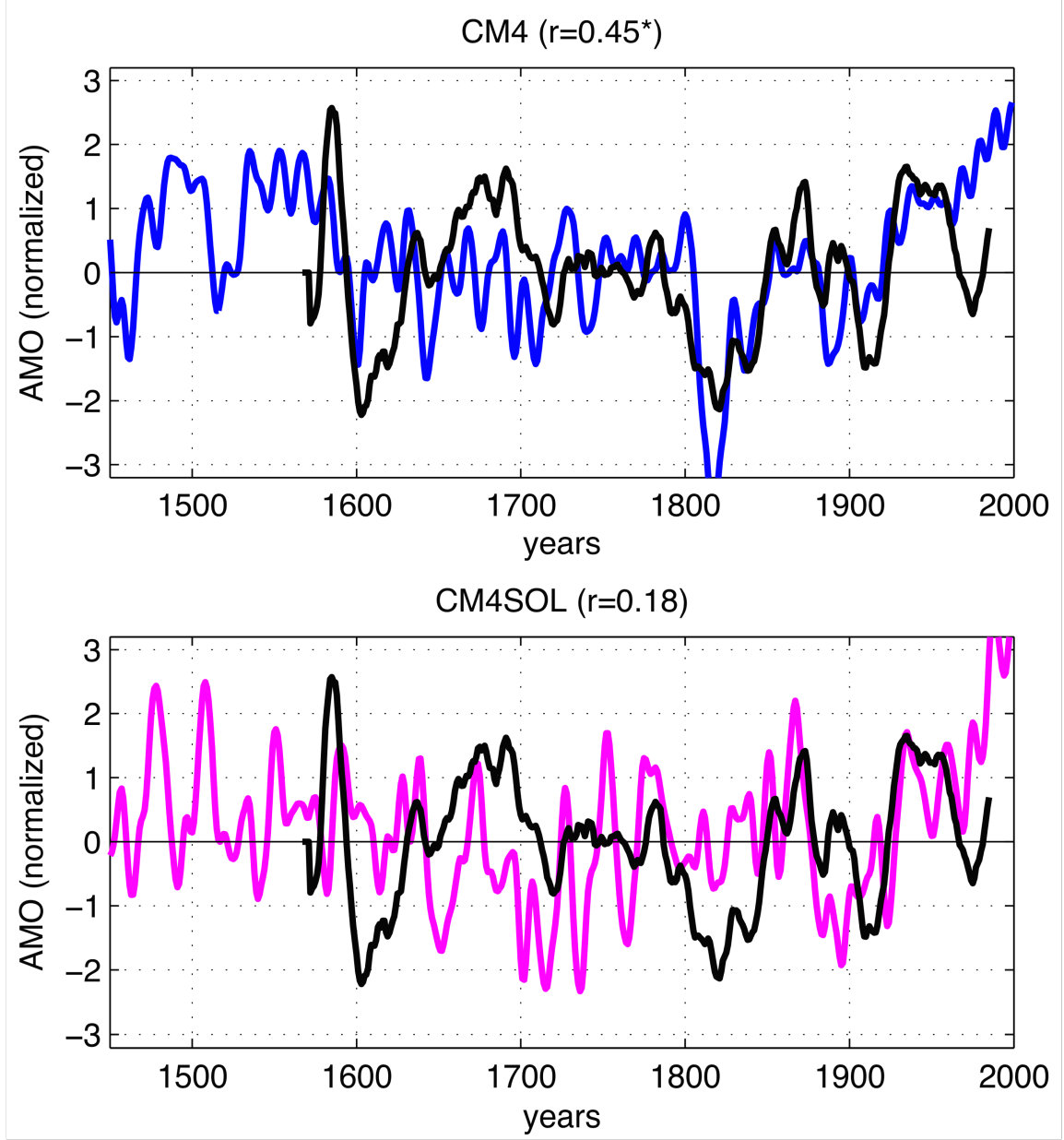

Figure 13: AMO evolution from the reconstruction of Gray et al. (2004) in black and in two IPSLCM4 simulations including before $1850 \mathrm{AD}$ a) solar and volcanic forcing (Mignot et al. 2011) and b) only solar forcing (Servonnat et al. 2010). From 1850 AD onwards, both simulations contain all observed forcings, including the growing increase in greenhouse gas concentrations. AMO is defined as the low pass filtered (10 years cutoff frequency) SST average from the Equator to $60^{\circ} \mathrm{N}$ in the Atlantic. For easier comparison, all time series have been normalized with respect to the full period covered by the reconstruction ([1576-1985]). 


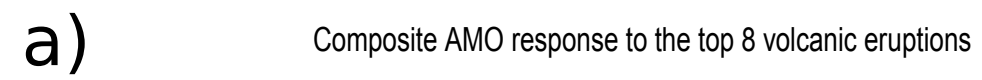

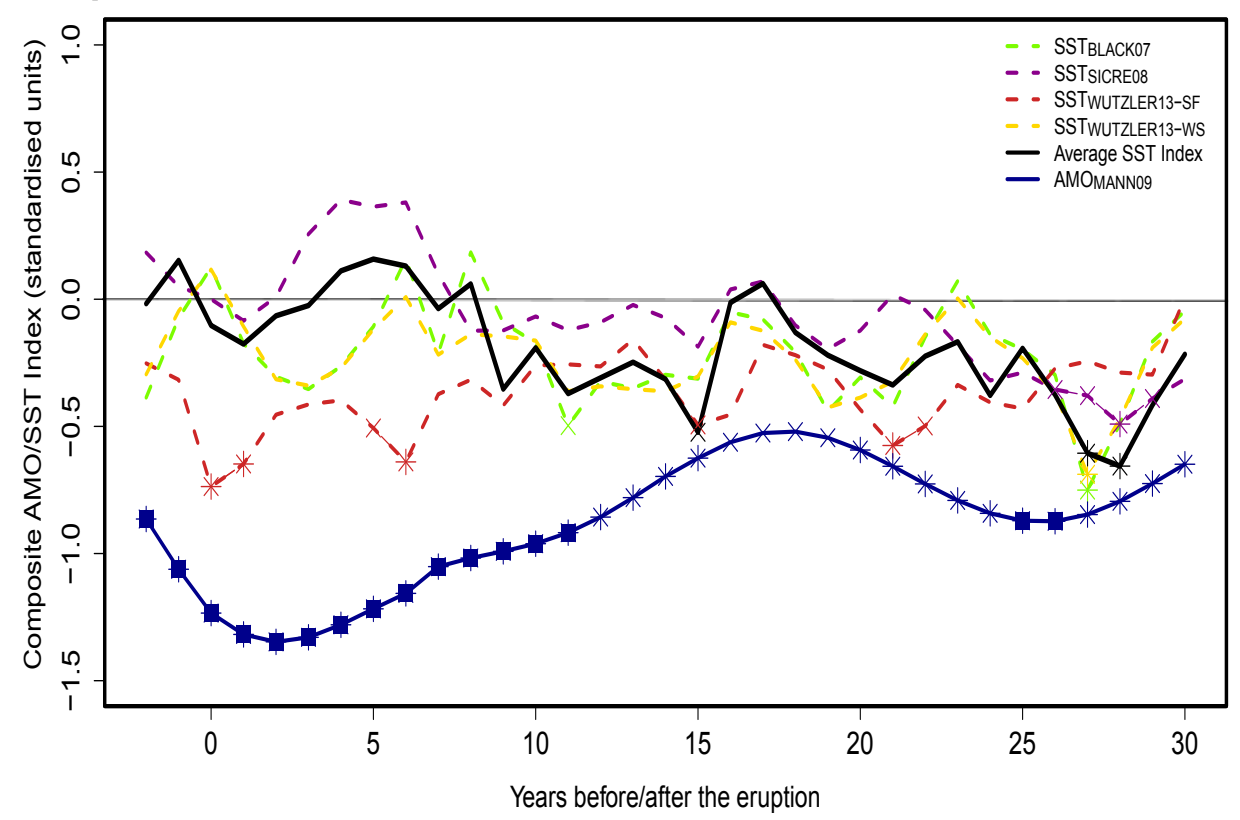

b) Composite AMO response to the top 8 volcanic eruptions

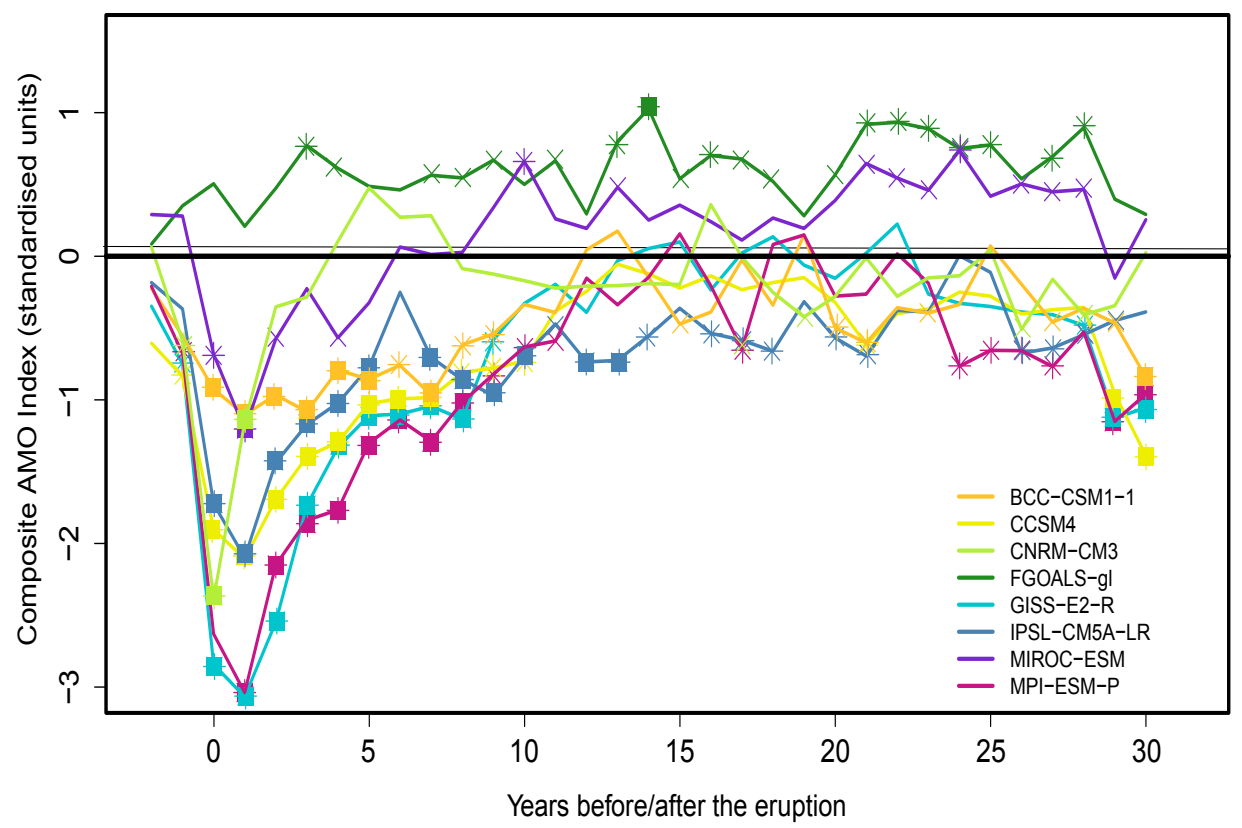

Figure 14: a) The same as in Figure 7a but for the AMO reconstruction (in standardized unit) from Mann et al (2009), and four other quasi-yearly resolved SST reconstructions from the North Atlantic $\left[0-60^{\circ} \mathrm{N}\right]$. b) The same as in Figure $7 \mathrm{~b}$ but for an AMO index (in standardized units) defined as the SST average over the North Atlantic. To allow for a direct comparison with the SST reconstructions, no detrending in the simulated AMO time series has been applied. All AMO time series are standardised as in Figure 7. 


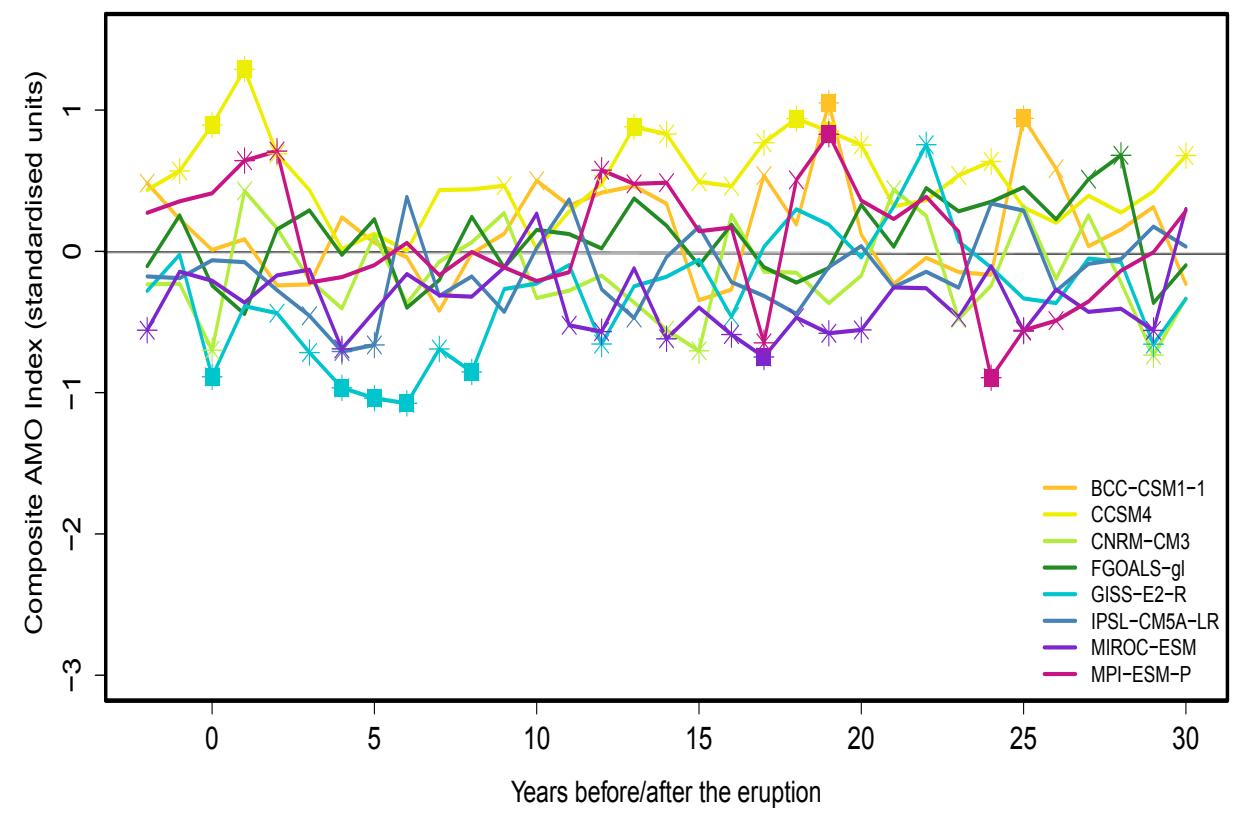

b) Composite AMOC response to the top 8 volcanic eruptions

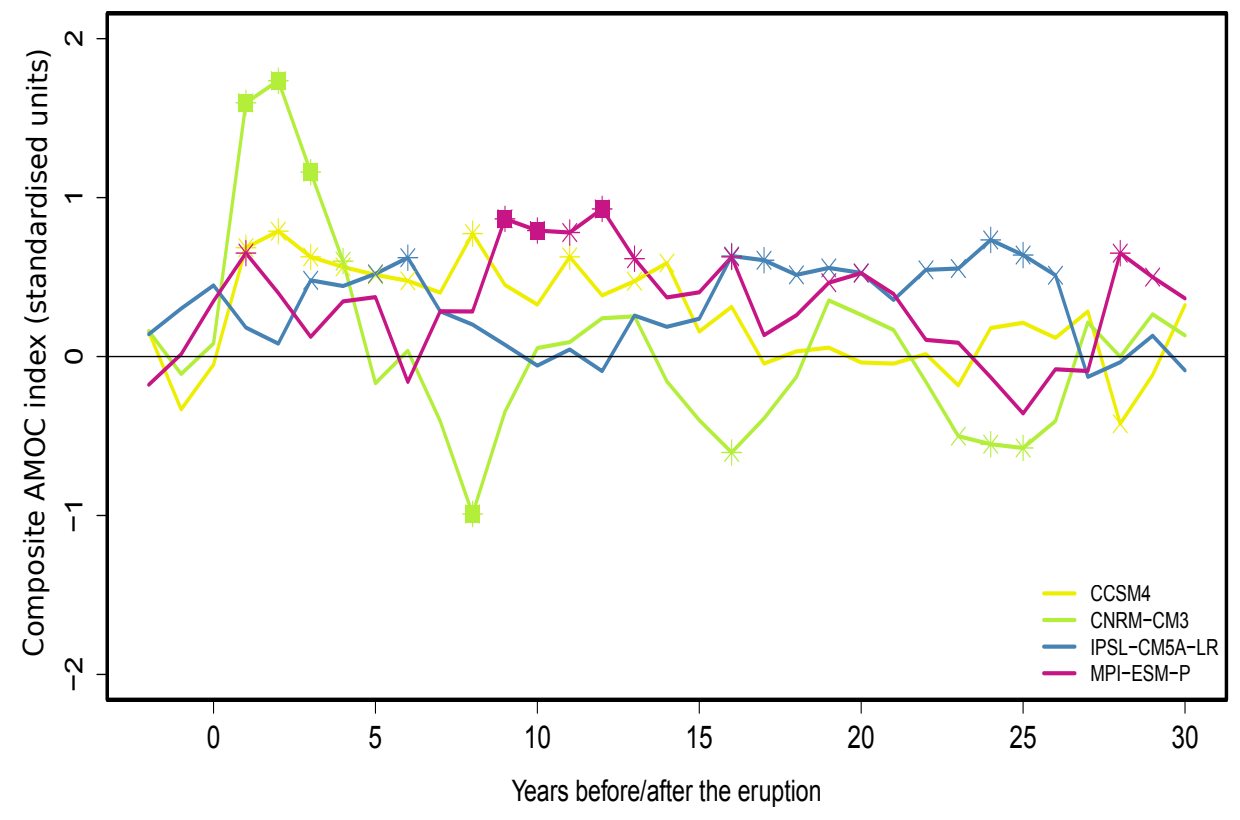

Figure 15 : a) The same as Figure 14b except that the AMO indices (in standardized unit) are now detrended following the definition in Trenberth \& Shea (2006). This consists in removing the global SST signal from the North Atlantic average. The idea behind is to remove the direct effect of the radiative forcings, and thus focus on the internally-driven variability specific to the Atlantic basin. ) Same as a) but for an AMOC index (in standardized units) defined as the maximum value of the Atlantic meridional streamfunction (only available for four models). All AMO and AMOC times series are standardised as in Figure 7. 

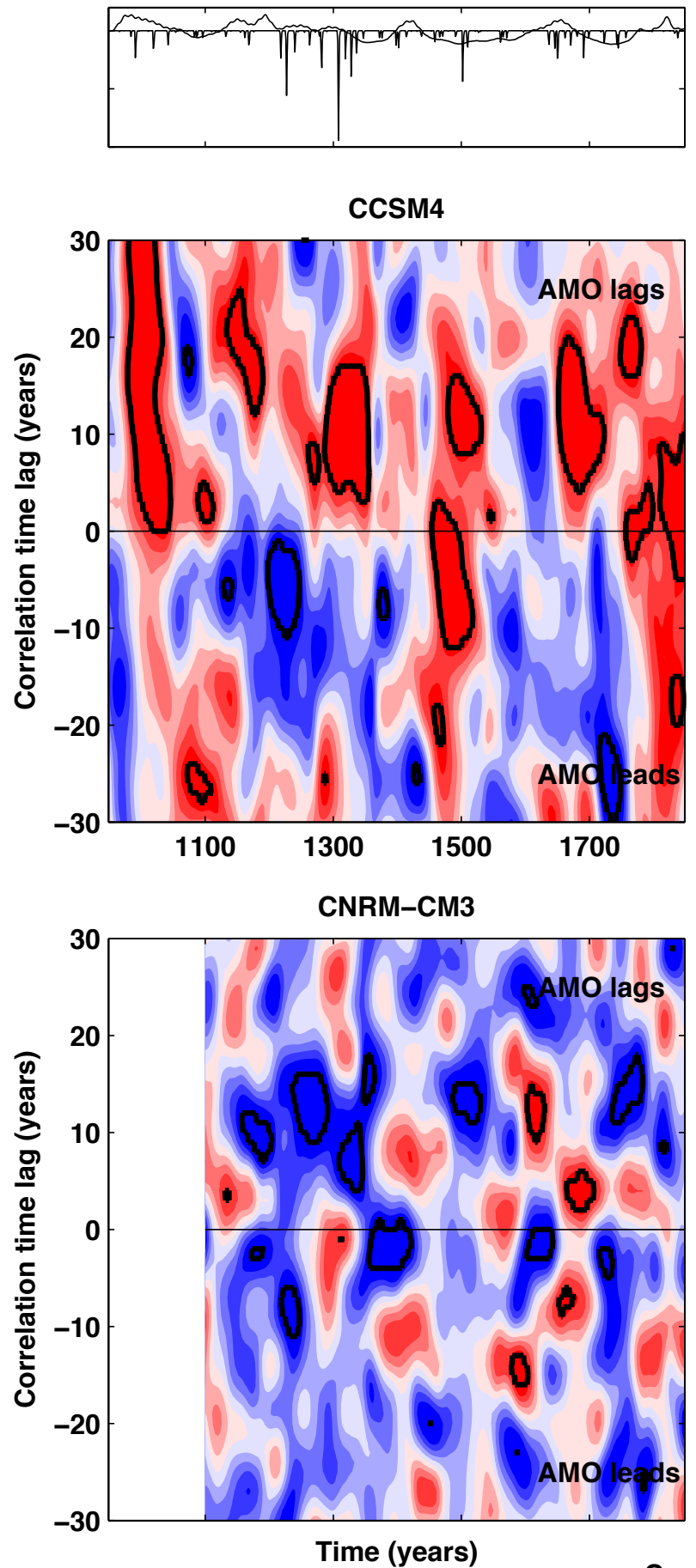

Correlation
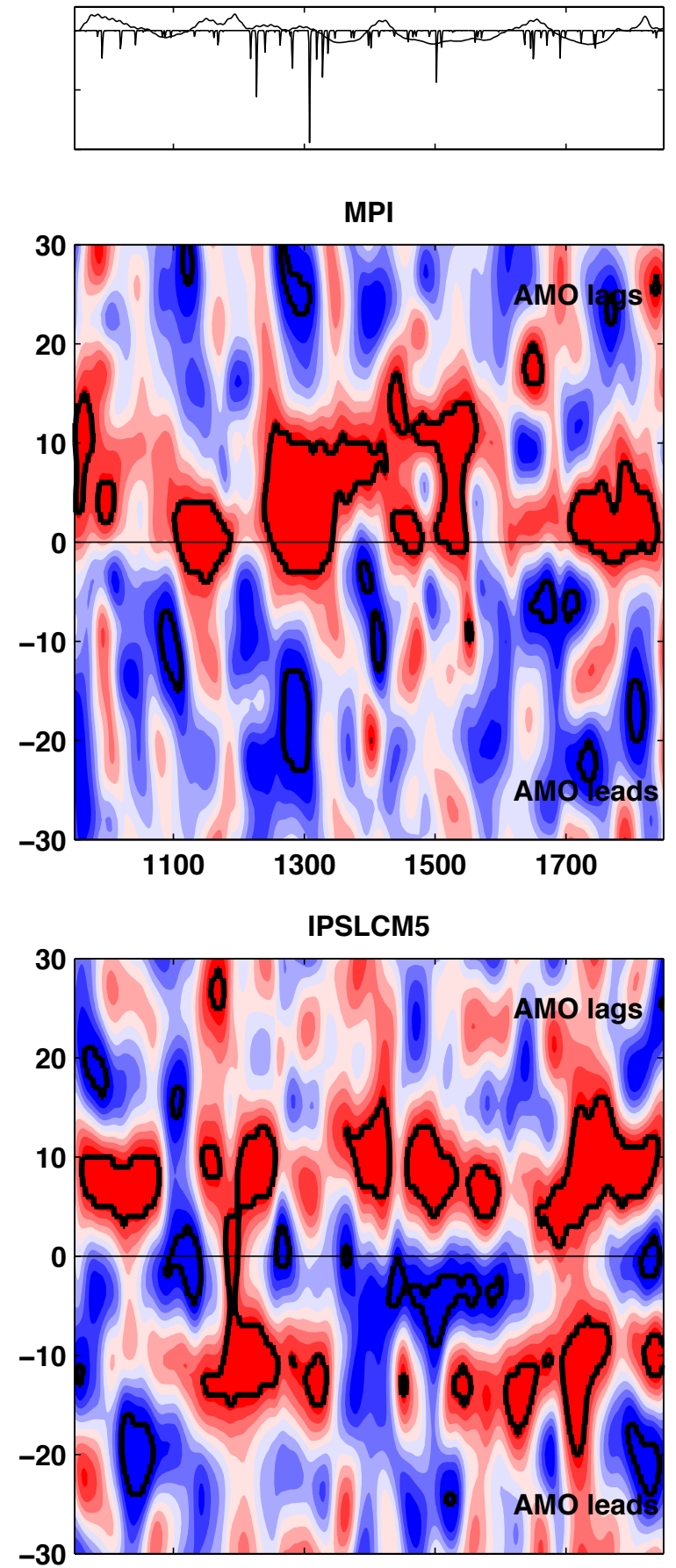

Time (years)

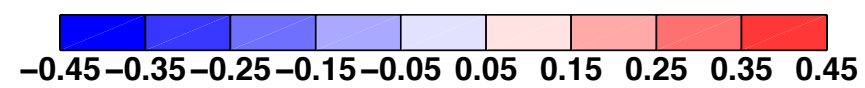

Figure 16: Cross-correlation between AMO and AMOC for 5 simulations of the last millennium, as a function of lags, in years (vertical axis). Correlations are computed over sliding tapered cosine windows of 100 years' width, and the horizontal gives the time in the middle of this interval. Black contours highlight significant correlations at the $95 \%$ level. The top of each panel shows the chronology of solar and volcanic forcing. 